\title{
Exploring the influence of organic species on pre- and post-nucleation calcium carbonate
}

\author{
Paolo Raiteri, ${ }^{a}$ Raffaella Demichelis, ${ }^{a}$ Julian D. Gale, ${ }^{\text {a** Matthias }}$ \\ Kellermeier, ${ }^{b}$ Denis Gebauer, ${ }^{b}$ David Quigley, ${ }^{c}$ Louise B. Wright ${ }^{d}$ \\ ${ }_{5}$ and Tiffany $R$. Walsh ${ }^{\text {e }}$ \\ DOI: 10.1039/b000000x [DO NOT ALTER/DELETE THIS TEXT]
}

Organic additives are well known to influence the nucleation and growth of minerals. A combination of experimental and theoretical methods has been used to probe how three simple additives, containing varying numbers of 10 carboxylate groups, influence the early stages of the growth of calcium carbonate. Computationally, the free energy landscape has been examined for each additive binding to $\mathrm{Ca}^{2+}$, the calcium carbonate ion pair, the surface of an amorphous calcium carbonate nanoparticle, and the basal plane of calcite. The different influence of the three organic ligands on the is early stages of growth of calcium carbonate observed experimentally can be rationalised in terms of the degree of association of each anion with the species present prior to, and immediately after nucleation.

\section{Introduction}

Calcium carbonate occurs naturally in the structures of many organisms as a result 20 of biomineralisation. ${ }^{1}$ Aside from playing either a protective role in shells ${ }^{2}$ or a support function in skeletons, ${ }^{3}$ it has also been found in primitive vision systems as an optical material. ${ }^{4}$ Unsurprisingly, this diversity of purpose and wide occurrence in nature has led to calcium carbonate being one of the most extensively studied substances in the field of biomineralisation. ${ }^{5,6}$ Despite this, there remains much to ${ }_{25}$ learn regarding the pathways by which organisms can selectively choose between polymorphs and organise crystallites into complex morphologies. ${ }^{7}$ Factors including the control of ion concentration, ${ }^{5}$ use of confined environments, ${ }^{8}$ and the interaction of organic species during crystal growth ${ }^{9}$ are all possible influences, though a clear picture of the atomic level detail remains elusive.

30 Even without the full complexity of an in vitro environment, understanding the crystallisation of calcium carbonate presents many challenges. Three crystalline polymorphs of $\mathrm{CaCO}_{3}$, namely calcite, aragonite and vaterite, in order of stability at ambient conditions, compete with each other, in addition to two hydrates (monohydrocalcite and ikaite) under certain conditions. ${ }^{10,11}$ While the structures of

35 calcite and aragonite are well known, the nature of the disorder within vaterite continues to be debated. ${ }^{12,13}$ Under conditions appropriate to biomineralisation there is increasing evidence that formation of crystalline polymorphs occurs via the initial nucleation of amorphous calcium carbonate (ACC), which subsequently transforms upon agglomeration of nanoparticles. ${ }^{6}$ ACC itself is a complex material that exhibits ${ }^{40}$ variable characteristics depending on whether it is biogenic or not. ${ }^{14}$ This may reflect varying degrees of water content from anhydrous to 1-2 waters per formula unit, ${ }^{15}$ the presence of impurities, most notably magnesium, ${ }^{16}$ but potentially even incorporation of organics, ${ }^{17}$ as well as perhaps intrinsic polyamorphism. ${ }^{18}$

[journal], [year], [vol], 00-00 | 1 
In the last few years it has become apparent that the fascinating growth mechanisms of calcium carbonate can challenge our understanding even prior to the appearance of ACC. In contravention of classical nucleation theory, the presence of stable pre-nucleation clusters beyond simple ion pairs can be detected ${ }_{5}$ experimentally. ${ }^{19}$ Through a combination of computer simulation and experiment, these precursor species have been shown to be a supramolecular polymer of calcium and carbonate ions that is constantly changing structure and remains in equilibrium with the solution, thereby avoiding a phase boundary. ${ }^{20}$ Furthermore, such clusters can change from rings to linear to branched chains all with a free energy difference

10 that is comparable to ambient thermal energy. Because of these characteristics, this new species has been named as a Dynamically Ordered Liquid Like Oxyanion Polymer (DOLLOP). The dominant coordination numbers for calcium and carbonate ions by other anions or cations, respectively, in DOLLOP are 1,2 and 3, thereby making it quite distinct from ACC. ${ }^{20,21}$

15 While the influence of the biological environment on ACC remains the focus of many studies, ${ }^{22}$ the identification of pre-nucleation species raises the possibility that the control of biomineralisation might begin even earlier in the crystal growth processes. ${ }^{23,24}$ Indications that this may be possible come from the prior discovery of Polymer-Induced Liquid-Precursor (PILP) ${ }^{6,25}$ Here the addition of polyaspartate to a 20 solution of calcium carbonate is found to generate a phase separated liquid mineral precursor whose shape can be manipulated. Inspired by this possibility, the aim of the present study is to perform a first examination of how the presence of organic species may alter the nature of pre-nucleation species of calcium carbonate. Rather than attempt to probe an in vitro system with all its inherent complexity, we begin 25 by exploring the effects of several simple organic additives under controlled conditions. Specifically, we consider the three anions of acetate $\left(\mathrm{CH}_{3} \mathrm{CO}_{2}{ }^{-}\right)$, aspartate $\left(\mathrm{O}_{2} \mathrm{CCH}\left(\mathrm{NH}_{3}{ }^{+}\right) \mathrm{CH}_{2} \mathrm{CO}_{2}{ }^{-}\right)$and citrate $\left({ }^{-} \mathrm{O} 2 \mathrm{C}(\mathrm{OH})\left(\mathrm{CH}_{2} \mathrm{CO}_{2}{ }^{-}\right)_{2}\right)$ that contain a varying number of carboxylate groups from one to three, as well as different net charges. It is hoped that this information may offer preliminary insights as to how

30 larger and more complex organics with similar functional groups may direct the nucleation and growth of calcium carbonate.

\section{Methodology}

\section{A Experimental}

All chemicals were used without further purification. Aqueous solutions were ${ }_{35}$ prepared with water taken from a Milli-Q system. Details regarding our $\mathrm{pH}$-constant experimentation have already been described extensively in the literature. ${ }^{19,23,26}$ However, we now utilize vessels that are surrounded by a jacket fed with oil from a thermostat, keeping the solution temperature constant at $(25 \pm 0.2)^{\circ} \mathrm{C}$. Titrations were carried out by dosing $10 \mathrm{~mm}$ calcium chloride solution ( $\mathrm{Ca}$ ion standard,

${ }_{40}$ Metrohm) into $50 \mathrm{~mL}$ of a $10 \mathrm{mM}$ carbonate buffer $(\mathrm{pH}=9.00$, prepared using sodium hydrogen carbonate from Riedel-de Haen (ACS reagent, $\geq 99.7 \%$ ) and anhydrous sodium carbonate from Sigma-Aldrich (ACS reagent, $\geq 99.9 \%$ )) at a constant rate of $10 \mu \mathrm{L} \mathrm{min}{ }^{-1}$, while the calcium potential was recorded with an ionselective electrode and the pre-set $\mathrm{pH}$ was kept constant by means of counter${ }_{45}$ titration with $10 \mathrm{mM} \mathrm{NaOH}$ (Alfa Aesar, standard solution). This procedure allows to determine the concentration of free calcium and carbonate ions throughout the different early stages of precipitation (i.e. pre-nucleation, nucleation, post-

2 | [journal], [year], [vol], 00-00 
nucleation). ${ }^{19,23}$ The reference experiment without additives was compared to assays in which $1 \mathrm{mM}, 5 \mathrm{mM}$, and $10 \mathrm{mM}$ of L-aspartic acid (Acros, $\geq 98 \%$ ) or sodium acetate (NaOAc, Merck, anhydrous, p.a.) were added to the carbonate buffer of the same $\mathrm{pH}$ prior to titration. Additive-containing buffers were prepared by mixing 20 ${ }_{5} \mathrm{mM}$ carbonate buffer with an equal volume of a 2,10 , or $20 \mathrm{~mm}$ solution of the respective additive in water, which had previously been adjusted to $\mathrm{pH} 9.00$ with $\mathrm{NaOH}$. For further comparison and to elucidate the role of ionic strength, additional reference runs were carried out in which carbonate buffers containing different amounts of sodium chloride (VWR Prolabo, AnalaR, $\geq 99.9 \%$ ) were used. Finally, in 10 order to assess binding of $\mathrm{Ca}^{2+}$ ions by the additives, independent titration experiments were conducted where $\mathrm{CaCl}_{2}$ was dosed into aqueous solutions of the additives (as well as $\mathrm{NaCl}$ ) at corresponding concentrations (likewise set to $\mathrm{pH}$ $9.00)$, but in the absence of carbonate.

\section{2. B Simulation methods}

In order to probe the atomistic detail of what is occurring when organic additives are present during the pre- and early post-nucleation period of calcium carbonate formation from aqueous solution we have performed molecular dynamics simulations based on force field methods.

20 The key to reliable simulations is the quality of the force field parameterisation. We have extensively studied this aspect in previous works, to ensure that important aspects of the thermochemistry of calcium carbonate are correctly described. ${ }^{27}$ In particular, a model has been developed that accurately reproduces the free energy difference between calcite and aragonite at ambient conditions, while also capturing

${ }_{25}$ the metastability of the disordered vaterite phase. Furthermore, the interactions of the component ions, $\mathrm{Ca}^{2+}$ and $\mathrm{CO}_{3}{ }^{2-}$, with water, as represented by the $\mathrm{SPC} / \mathrm{Fw}$ force field, ${ }^{28}$ have been fitted in order to reproduce experimental free energies of solvation of the species. Here we adopt the latest version of the force field that includes anharmonic bond-bond and bond-angle coupling within the carbonate anion. A full ${ }_{30}$ description of the force field, including the parameters can be found elsewhere. ${ }^{20} \mathrm{By}$ combining the quasiharmonic lattice free energy with the solvation free energies of the ions, as well as correcting for the gas phase translational and rotational entropy (for carbonate) of the ions, the free energy of dissolution can be estimated. Here our computed value for calcite of $+46.3 \mathrm{~kJ} / \mathrm{mol}$ compares favourably with the 35 experimental value of $+48.4 \mathrm{~kJ} / \mathrm{mol}$.

In the present work it was necessary to extend the parameterisation of the force field to include organic species and their interaction with $\mathrm{Ca}^{2+}$ and the other species present in solution. There are many different organic force fields already available from the biological community and there have already been numerous works that 40 have used several of these models to consider the interaction of organic molecules with calcium carbonate. One of the earliest was that of Griffiths and Heyes ${ }^{29}$ who used the CVFF force field ${ }^{30}$ to study the binding of sulfonates and phenates to small amorphous $\mathrm{CaCO}_{3}$ clusters. Later de Leeuw and $\mathrm{Cooper}^{31}$ also employed the same organic force field to examine the binding of small $\mathrm{C} 1 \& \mathrm{C} 2$ organics on calcite,

${ }_{45}$ though in combination with a shell model for water that was subsequently found to freeze at longer timescales. Recently Aschauer et $a l^{32}$ have extended this combined shell model water approach to the study of polyacrylic and polyaspartic acids on calcite, but now using Dreiding ${ }^{33}$ instead of CVFF. Duffy, Harding and co-workers

[journal], [year], [vol], 00-00 | 3 
have examined the influence of self-assembled monolayers on the nucleation and growth of calcium carbonate ${ }^{34,35}$, in particular for stearic acid, using the CHARMM $^{36}$ force field for the organic part. A recent study of the binding of alkaline metal cations to the aspartate anion by Hamm et $a l^{37}$ also favours the use of ${ }_{5}$ CHARMM for the organic interactions. In contrast, Metzler et $a l^{38}$ have used the $\mathrm{AMBER}^{39}$ force field to examine peptide-induced aggregation of calcium and carbonate ions in solution. An attempt to produce a general force field for the simulation of biomineralisation was made by Freeman et $a l^{40}$ based on the $\mathrm{CaCO}_{3}$ model of Pavese and co-workers ${ }^{41}$ combined again with the use of AMBER for the 10 organic part. Surveying the trends in the above literature, it is clear that the trend is towards the use of more extensive and specific organic force fields, such as AMBER and CHARMM, as opposed to more generic parameterisations like CVFF and Dreiding. Here we choose to adopt the CHARMM force field for the intramolecular organic interactions.

15 When considering the organic-water interactions it is necessary to refit the interaction parameters, rather than taking them unmodified from the existing CHARMM force field, for two reasons. Firstly, we are using the SPC/Fw force field for water, whereas CHARMM parameters were derived for the TIP3P water model. Secondly, an important part of our philosophy is to try to ensure that the 20 experimental thermodynamics are reproduced where possible, and in particular, the free energy of solvation of all ions. Failure to consider this may lead to incorrect binding energies in the simulations. Hence the organic-water parameters have been varied to ensure the solvation properties are correct (final parameters are given in the Electronic Supplementary Information as Tables S1-3).

25 One of the issues that must be addressed in the above approach is to determine the reference value of the free energy of solvation. While there are numerous experimental and theoretical studies available for the solvation of acetate anion, values for L-aspartate and citrate in the protonation state appropriate to the $\mathrm{pH}$ of interest are harder to find. In order to supplement the experimental information 30 available, which will be discussed during the results section, we have also performed quantum mechanical calculations to estimate the free energy of solvation. Here the relevant anions were optimised using density functional theory with the M06 meta hybrid exchange-correlation functional of Zhao and Truhlar, ${ }^{42}$ in conjunction with a $6-31+G^{* *}$ Gaussian basis set. These calculations were performed within the ${ }_{35}$ framework of the $\mathrm{SM}^{43}$ implicit solvation model with the parameters, including dielectric constant, set appropriate to water. All calculations have been performed using the QChem software. ${ }^{44}$ While inclusion of an explicit solvation shell of water is often required in order to obtain an accurate description of species in solution, the SM8 model has been calibrated to yield accurate solvation free energies without 40 this.

Interactions between the organics and water were initially taken from the bicarbonate-water interactions taken from our previous study with rescaling of the $A$ parameter by the ratio of the charges. The parameters for the water-carboxylate interactions were then refined against the target free energy of solvation. All initial

${ }_{45}$ free energies of solvation were computed using a modified version of DL_POLY_2.19 $9^{45,46}$ by use of a two-stage perturbation approach, as described in previous work. Subsequent refinement was performed by using the perturbation between two sets of interatomic parameters to compute the change in the free energy of solvation.

4 | [journal], [year], [vol], 00-00

This journal is (? The Royal Society of Chemistry [year] 
The final set of force field parameters required for the present work involves the interaction between $\mathrm{Ca}^{2+}$ and the organic species. Given that there is limited crystallographic or other experimental evidence to fit against, we have taken a similar approach to previous works by using scaling relationships to determine the ${ }_{5}$ coefficients for a Buckingham potential in the case of the $\mathrm{Ca}-\mathrm{O}$ interaction:

$$
U_{i j}=A_{i j} \exp \left(-\frac{r_{i j}}{\rho_{i j}}\right)-\frac{C_{i j}}{r_{i j}^{6}}
$$

Here the leading coefficient is scaled by the charge of the oxygen with which the calcium is interacting (given that the calcium charge is fixed at +2 ) relative to the parameter fitted for $\mathrm{Ca}-\mathrm{O}$ in bulk calcium carbonate:

$$
A_{i j}=A_{i j}^{C a-C O_{3}}\left(\frac{q_{O}}{q_{O\left(\mathrm{CO}_{3}\right)}}\right)
$$

While this scaling relationship was found to work well in transferring parameters from carbonate to bicarbonate, evaluation of the ion pair free energies based on the resulting short-range potentials showed a systematic over-binding. To correct for this, a uniform scaling was applied of 1.34 to the Buckingham $A$ parameters between 15 calcium and carboxylate oxygen. The final set of intermolecular force field parameters used in the present work, including those previously published for aqueous calcium carbonate systems, are given as Electronic Supporting Information (Tables S1-S3 with Figures S1-S3 showing the charges used). Intramolecular interactions are generated from the CHARMM force field ${ }^{47}$ using the CGenFF ${ }_{20}$ program. ${ }^{48}$ Note that long-range interactions, including Coulomb terms, are excluded for 1-2 and 1-3 connected atoms, with the exception of bicarbonate and acetate, where the 1-4 interactions were also excluded.

One of the issues that must be addressed when simulating carbonate speciation in water, as well as that of polybasic organics, is that of $\mathrm{pH}$. The $\mathrm{pKa}$ of acetic acid is ${ }_{25} 4.76$ and so we can consider it as being essentially fully dissociated under the basic conditions of interest here. Aspartic acid has three pKa values of approximately 2.1, 3.9 and 9.8 and so will have lost both carboxyl protons under mildly basic conditions. Hence we consider aspartate as being in the zwitterionic form in which the amine group remains protonated to give a net charge of -1 . Citric acid is also 30 tribasic, but with pKa values of 3.2, 4.8 and 5.2 the citrate anion can be regarded as completely deprotonated under relevant conditions.

In the conventional molecular mechanics force field derived above the protonation state of all functional groups must be specified a priori and thus this represents an approximation. In order to test the validity of the assumptions made 35 we have also chosen to perform simulations using a reactive force field model. Here we use the ReaxFF formalism of van Duin and co-workers ${ }^{49}$ as implemented in the program GULP. ${ }^{50}$ Parameters for aqueous calcium carbonate systems in ReaxFF have been determined by Gale et $a l^{51}$ using a fixed charged model for the calcium cation, which essentially remains fully ionic in aqueous conditions. A fully reactive ${ }_{40}$ model for calcium-containing systems has subsequently been derived, ${ }^{52}$ though this appears not to be offer substantial benefits for the present system and yields inferior results for calcite. Thus the unreactive calcium model is retained in the present

[journal], [year], [vol], 00-00 | 5 
study. To allow for the incorporation of organics the parameters developed for the simulation of peptides ${ }^{53}$ in water have been used.

All molecular dynamics simulations using ReaxFF have been performed using a domain decomposition algorithm with iterative charge solution. A time step of $0.5 \mathrm{fs}$ 5 was employed in combination with a stochastic integrator. ${ }^{54,55}$ For each combination of ions studied, the species were placed in a system containing 308 water molecules within a cubic cell of just over $20 \AA$ along each side. An NPT ensemble was used with the cubic cell being allowed to vary isotropically at $298.15 \mathrm{~K}$. Where the system was charged, a neutralising background charge was applied, as well as a 10 Madelung correction, to remove the spurious interaction between images. All simulations were run for 100 ps of equilibration followed by $2.5 \mathrm{~ns}$ for production.

All MD simulations using the standard unreactive force field approach have been performed with LAMMPS $^{56}$ (version as of $27^{\text {th }}$ October 2011) using a 1 fs time step and the PPPM algorithm for the calculation of the reciprocal space contribution to

15 the electrostatics. A chain of 5 Nosé-Hoover thermostats and barostats with relaxation times of 0.1 and $1.0 \mathrm{fs}$, respectively, has been used in all simulations to control temperature and pressure ensuring that the correct ensemble was sampled. The free energy calculations have been performed using the PLUMED 1.3 plug-in ${ }^{57}$ in the $N V T$ ensemble. The distance, or minimum distance, was used as a collective 20 variable, depending on the number of carboxyl groups that were present in the binding species. Well-tempered metadynamics ${ }^{58}$ with a bias factor equal to 11 and 50 multiple-walkers ${ }^{59}$ have been used in all calculations. The initial Gaussian height and width were $k_{B} T$ and $0.1 \AA$, respectively, with a new Gaussian being added every $0.5 \mathrm{ps}$ during a total simulation length of $130 \mathrm{~ns}$.

25 Car-Parrinello molecular dynamics (CPMD) simulations of the citrate-water system were also carried out to provide additional points of comparison for the force fields developed in this work. Due to the computational expense inherent to firstprinciples simulations, we considered only modest length-scales and time-scales here, with a system comprising one citrate molecule (featuring three carboxylate 30 anionic groups), 128 water molecules, and three $\mathrm{Na}^{+}$counter ions (405 atoms in total). To perform these simulations we used the CPMD software package ${ }^{60}$ version 3.13.2, with the BLYP ${ }^{61,62}$ functional and ultrasoft ${ }^{63}$ pseudopotentials, along with a plane wave cut-off of $340.1 \mathrm{eV}$. The system was contained in a cubic simulation cell of side-length $16.1 \AA$, with 3 -D periodic boundary conditions used throughout. Prior 35 to the CPMD run, the system was geometry optimised to a threshold of $2.57 \mathrm{eV}^{-1}$. All atoms were free to move in the simulation, which was conducted in the NVT ensemble at $300 \mathrm{~K}$, using a Nosé-Hoover thermostat with a coupling-constant of $2915 \mathrm{~cm}^{-1}$. A time-step of $0.0968 \mathrm{fs}$ and a fictitious electron mass of 400 atomic units were used, and $k$-point sampling included the $\Gamma$-point only. The duration of the ${ }_{40}$ CPMD simulation was 5 ps in total, of which the first 2 ps was treated as equilibration and only the last 3 ps was used for subsequent analysis.

For the purposes of our analysis of the first principles molecular dynamics, we define two types of carboxylate present in citrate, denoted as OM and OE, signifying the central and end carboxylates respectively, with the label $\mathrm{OH}$ indicating the 45 oxygen of the central hydroxyl group. We have calculated radial distribution functions (RDFs) around the $\mathrm{OM}, \mathrm{OE}$ and $\mathrm{OH}$ sites using a bin width of $0.02 \AA$.

6 | [journal], [year], [vol], 00-00 


\section{Results}

\section{A Pre-nucleation clusters in the absence of organics}

The nature of pre-nucleation clusters in the absence of additives other than a background electrolyte has already been previously described in earlier work. ${ }^{20}$ Here 5 we describe the salient features that are relevant to any discussion of the influence of additives. In our previous studies we have demonstrated that calcium and carbonate ions come together in solution to create both ion pairs and higher aggregates known as DOLLOP. These DOLLOP structures involve dynamically evolving chains of alternating calcium and carbonate ions that can branch, form rings, lose and gain 10 ions. Because of the rapid exchange of ions with solution there is no phase boundary between these species. The combination of strong hydration, leading to enthalpic stabilisation, and favourable entropic contribution, resulting from the large number of microstates available within an almost degenerate energy, means that these species are thermodynamically stable with respect to ions and ion pairs in solution at

$15 \mathrm{mM}$ concentrations and alkaline $\mathrm{pH}$. The DOLLOP species remain structurally distinct from ACC, including hydrous ACC; whether there is an activated process that connects DOLLOP to ACC or whether conversion occurs by dissolution and regrowth remains unknown at present.

\section{3. B Solvation of isolated organic anions}

Following the approach taken for the derivation of a force field model to describe both $\mathrm{Ca}^{2+}$ and $\mathrm{CO}_{3}{ }^{2-}$ in aqueous solution, the interaction of the three organic anions with water has been parameterised in order to reproduce the free energy of hydration, while also trying to ensure a reasonable solvation environment about the ${ }_{25} \mathrm{key}$ functional groups. Any error in the thermodynamics of solvation is likely to impact on the competition between the anions binding to cationic species versus remaining isolated in aqueous solution.

In the case of acetate there are numerous values for the free energy of hydration available in the literature, as summarised in Table 1. Because the experimental 30 values for this quantity can only be inferred indirectly, in the case of an anion via a thermodynamic cycle, this leads to a degree of variability. Theoretically, there is also some scatter in the values with Gao et $a l^{64}$ demonstrating that the value obtained from ab initio calculations is sensitive to the combination of the level of quantum mechanical theory, basis set and solvation model chosen. Similarly within 35 force field studies the results will depend on the parameterisation of the water model and whether polarisation effects are included. ${ }^{65}$ Here the value for the free energy of hydration from the model adopted spans the range of several of the less extreme values, to within the statistical uncertainty in the final result, while being slightly less exothermic than the value obtained from the quantum mechanical calculations 40 performed in the present work.

There are also many studies of the aspartate anion in the literature because of its relationship to biological molecules. This includes examinations of conformational and tautomeric equilibria, ${ }^{66}$ and calculation of the $\mathrm{pK}_{\mathrm{a}}$ values. ${ }^{67}$ Despite this, there appears to be no explicit estimate of the free energy of hydration available. ${ }_{45}$ Furthermore, because the zwitterionic configuration that is adopted by the anion in solution is unstable in the gas phase, it is not possible to construct a purely experimental thermodynamic cycle to obtain the free energy of hydration.

[journal], [year], [vol], 00-00|7 
Consequently we have adopted the SM8/M06/6-31+G** value for this quantity as an appropriate target. Although it would be possible to precisely reproduce the quantum mechanical free energy of solvation by fine-tuning of the water interactions, it was considered that a slight overestimate was acceptable given the uncertainties in the $s$ target value and that the acetate value also deviates from the quantum mechanical estimate in the same direction.

For both citric acid and citrate there is also a dearth of information in the literature regarding the thermodynamics of hydration, again leading us to take the SM8/M06/6-31+G** value as the best available estimate for evaluation of the force 10 field. As per acetate and aspartate, the absolute magnitude of the solvation free energy with the force field model is slightly under-estimated, but only by a few $\mathrm{kJ} / \mathrm{mol}$, which is well within the uncertainty of the target quantity.

Table 1 Comparison of literature values (experiment and theory) for the free energies of hydration 15 of the acetate, aspartate and citrate anions with the computed values at the M06/6-31+G** level of theory within the SM8 solvation model (QM) and force field calculations, as determined in the present work. All values are in $\mathrm{kJ} / \mathrm{mol}$.

\begin{tabular}{|c|c|c|c|}
\hline \multirow{4}{*}{ Experiment } & Acetate & Aspartate & Citrate \\
\hline & $-337.4^{\mathrm{a}}$ & & \\
\hline & $-322.2^{\mathrm{b}}$ & & \\
\hline & $-313.8^{\mathrm{c}}$ & & \\
\hline \multirow[t]{5}{*}{ Theory } & $-343.8^{\mathrm{a}}$ & & \\
\hline & $-341^{\mathrm{d}}$ & & \\
\hline & -278 to $-338^{e}$ & & \\
\hline & -320 to $-325^{\mathrm{b}}$ & & \\
\hline & $-324.3^{\mathrm{f}}$ & & \\
\hline QM (this work) & -334.4 & -418.9 & -1774 \\
\hline Force field (this work) & $-326 \pm 3$ & $-414 \pm 3$ & $-1771 \pm 3$ \\
\hline from $\operatorname{Ref}^{68} \mathrm{~b}$. from Re & from Ref. $^{70} \mathrm{~d}$. & from Ref & \\
\hline
\end{tabular}

20 Aside from considering the thermodynamics of solvation, it is also important to examine whether the structure of water around the organics is reasonable. While both acetate and aspartate have been studied previously in the literature, the least well-calibrated system is that of citrate. Given that it is the most highly charged of the three anions, it is also represents the most severe test of the degree of water 25 ordering in the first hydration shell. As there is no experimental data to compare against, that we are aware of, first principles simulations of citrate in water have been performed to provide comparative information. Sodium counter-ions were employed for charge neutrality as they have a low tendency to associate with the citrate anion. It is important to note that there are limitations on the interpretation of 30 the data obtained from the first principles molecular dynamics too, including the systematic errors of current GGA functionals for the structure and dynamics of water, and also the short time scale that is accessible. We note that inclusion of van der Waal's interactions appears to improve some aspects of the description of water, ${ }^{72}$ and artificially increasing the temperature may be a pragmatic approach to ${ }_{35}$ improve the results. However, neither of these approaches was used in the present work.

8 | [journal], [year], [vol], 00-00

This journal is @ The Royal Society of Chemistry [year] 

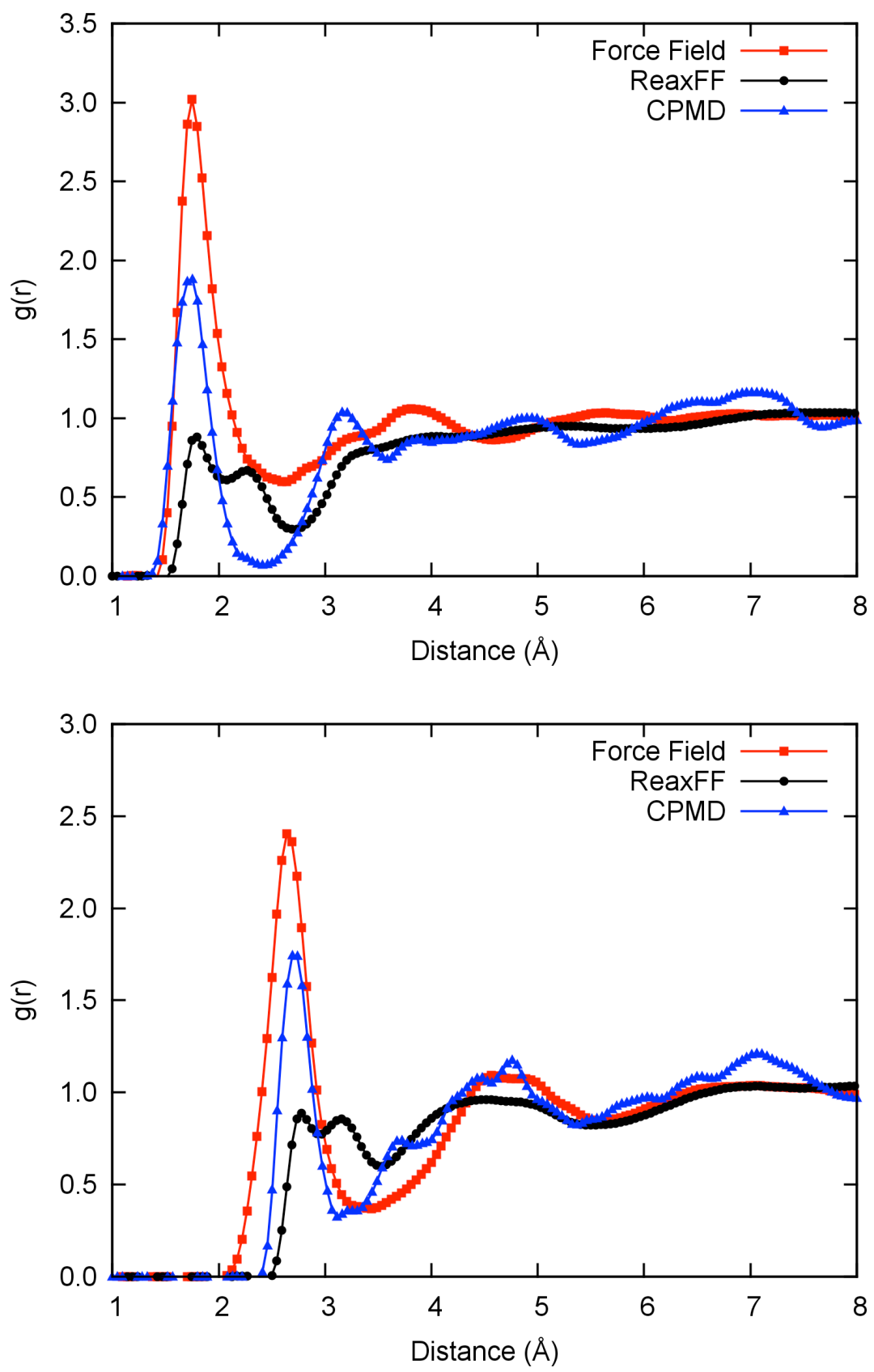

Figure 1 Radial distribution function $(\mathrm{g}(\mathrm{r}))$ for the oxygens $(\mathrm{OE})$ of the two equivalent carboxylate groups of citrate to $\mathrm{H}$ (top) and $\mathrm{O}$ (bottom) of water.

In Figure 1 the radial distribution functions for selected interactions between citrate and water are given, as determined according to the conventional force field, ReaxFF and first principles molecular dynamics (CPMD). Starting with the carboxylate groups, all three methods show good agreement as to the position of the

[journal], [year], [vol], 00-00 | 9 
first peaks for OE to both the hydrogen and oxygen of water. Even the subsequent solvation shells seen in the OE-OW distribution are in good agreement, allowing for the inherent noise present in the CPMD data due to the restricted sampling. The main discrepancy is that the ReaxFF model indicates some splitting of the first peak ${ }_{5}$ for both OE-OW and OE-HW, while this is absent for the other two methods. This is due to the fact that the ReaxFF citrate RDFs are computed from a simulation that also contains a $\mathrm{Ca}^{2+}$ counter ion. Although this cation is initially separated by half of a box length, during the course of the $2.5 \mathrm{~ns}$ run the ions diffuse together to form a solvent separated ion pair, thereby perturbing the distribution of hydrogen bonds 10 around citrate.

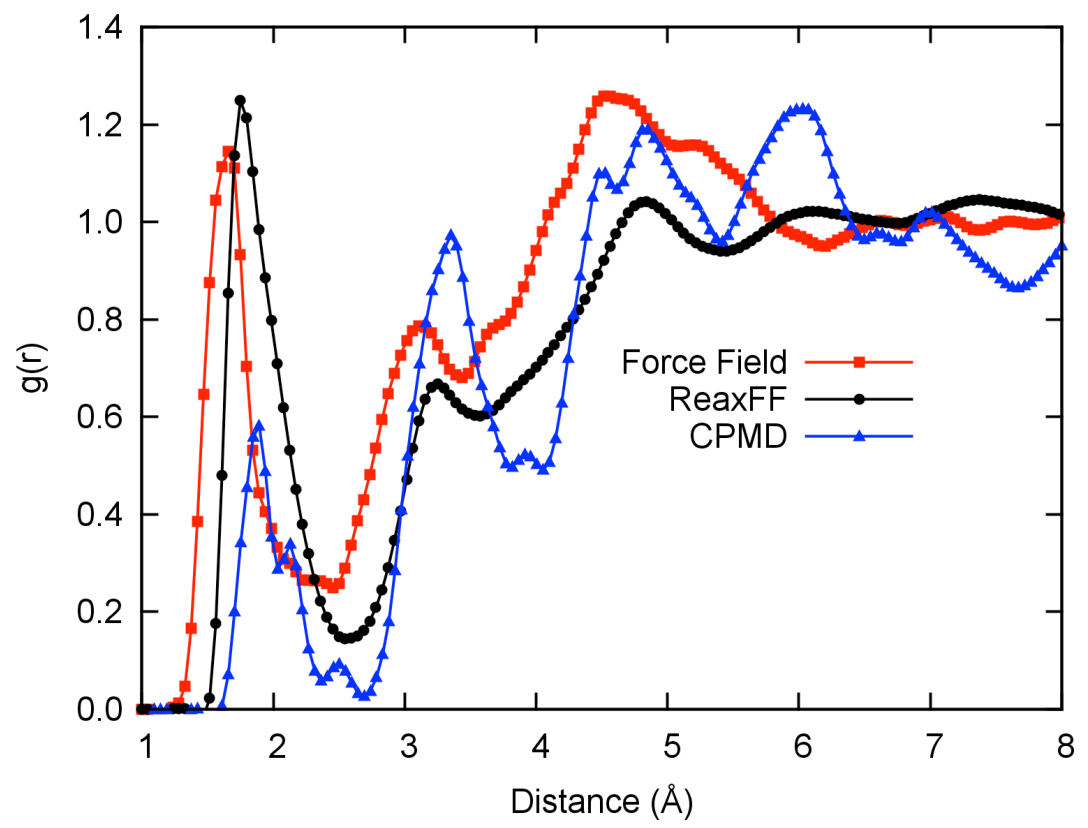

Figure 2 Radial distribution function for oxygen of the hydroxyl group of the citrate anion to the hydrogens of water molecules as computed with three different methods.

Turning to consider the radial distribution functions for the hydroxyl group of 15 citrate, as shown in Figure 2, here the different techniques are less in accord. In comparison to the first principles data, the hydroxyl group of citrate interacts too strongly with water as an acceptor of hydrogen bonds. However, the aforementioned caveats must be borne in mind before deciding where the correct result lies. In particular, it was observed in the ReaxFF simulations that an intramolecular 20 hydrogen bond between the $\mathrm{OH}$ and a carboxylate group of citrate could persist for considerably longer than the time scale of the first principles run. Consequently the initial configuration will influence the outcome, as there is insufficient time to equilibrate the intra- and inter-molecular configurations with the proper statistical weight. Given that the first principles dynamics starts from a conformation that has ${ }_{25}$ an intramolecular hydrogen bond, which lasts for $85 \%$ of the production phase, while the conventional force field shows little or no evidence of the same interaction in the long time limit, the deviations in the radial distribution function can be apportioned to the different conformations being sampled.

10 | [journal], [year], [vol], 00-00 


\section{C Influence of organics on pre-nucleation clusters}

Having calibrated the free energies of hydration for acetate, aspartate and citrate, we now turn to consider the simulation of the association between these anions and 5 species that would be present in solution prior to nucleation. In particular, the ion pairing between $\mathrm{Ca}^{2+}$ and the above organics is likely to be the initial mode of association:

$$
C a_{(a q)}^{2+}+X_{(a q)}^{n-} \Leftrightarrow \operatorname{CaX}_{(a q)}^{(2-n)+}
$$

Using metadynamics, we have computed the free energy profiles for each of the 10 above ion pairing reactions, as shown in Figure 3. The overall free energies of binding, after correction for the radial dependence of the entropy, are given in Table 2 along with literature values.

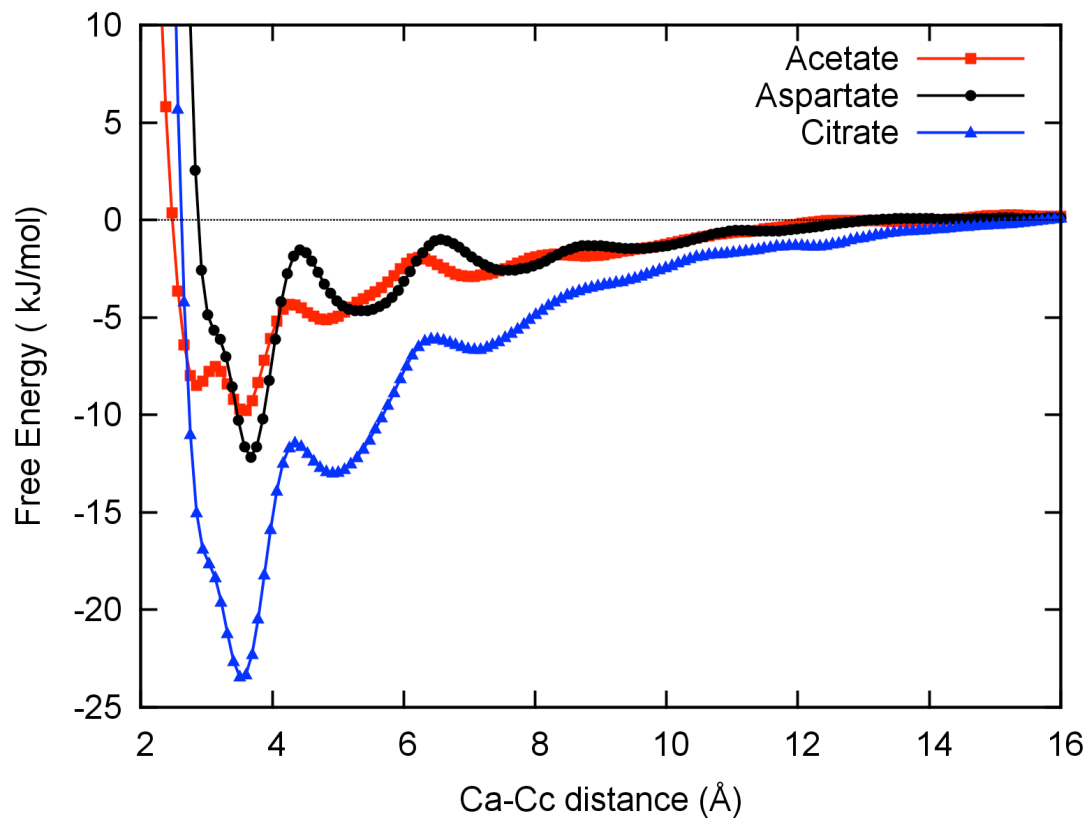

15 Figure 3 Free energy as a function of the minimum distance between calcium $(\mathrm{Ca})$ and the nearest carbon of any carboxylate group $(\mathrm{Cc})$ of the organic anions, acetate, aspartate and citrate, in aqueous solution. The zero of free energy is specified with reference to the fully separated ions.

It should be noted that computed values are in effect for the system at high dilution since the difference is taken between the free energy minimum and the 20 asymptotic limit for long-range separation. Under standard conditions of $1 \mathrm{M}$ the average separation between ions is reduced and so taking the commensurate free energy at this distance reduces the binding strength by up to $5 \mathrm{~kJ} / \mathrm{mol}$, depending on the species. Hence the discrepancy with the experimental values is actually less than that apparent from Table 2 when this is kept in mind. In the case of citrate we note ${ }_{25}$ that the interaction with $\mathrm{Ca}^{2+}$ has a longer range than for the other organics and so

[journal], [year], [vol], 00-00 | 11 
the free energy curve has not quite reached the plateau by the maximum distance sampled. However, the error is comparable with the statistical uncertainty.

Table 2 Comparison of literature values (experiment and simulation) for the free energies of ion pairing of calcium with the acetate, aspartate and citrate anions with the computed values from force 5 field simulation in the present work. Experimental values for acetate and aspartate/citrate are taken from ref. $^{73}$ and ref. ${ }^{74}$, respectively. All values are in $\mathrm{kJ} / \mathrm{mol}$.

\begin{tabular}{cccc}
\hline & Acetate & Aspartate & Citrate \\
Experiment & -4.4 to -7.1 & -6.7 & -19.0 \\
Previous simulation & & -16.7 & \\
Simulation & -9.5 & -12.2 & -20.7 \\
\hline
\end{tabular}

As initially computed with the straight scaling relationship for the repulsive Ca-O 10 interaction, all of the ion pairs were found to be over bound by a significant amount. Consequently, a single scaling factor of 1.34 was introduced for the Buckingham $A$ parameter of this interaction. Following this modification the general trend in the ion pairing free energies was found to be in good agreement with experiment, with citrate being the most strongly bound by a significant margin. Whether or not acetate 15 and aspartate have the correct relative order depends on the choice of experimental value, but the upper bound to the ion pairing free energy would be most consistent with the simulation data. In terms of the absolute free energy values, once the correction for the concentration back to standard conditions is allowed for, all of the simulation values are within ambient thermal energy from experiment. It should be 20 noted that although the well-tempered metadynamics is converged to $\sim 0.001 \mathrm{~kJ} / \mathrm{mol}$, the remaining discrepancy in the absolute values is hard to remove since it is within the residual statistical uncertainty due to fluctuations.

Although the above results justify the introduction of a modified scaling procedure, there is a risk that fitting of free energy differences for solution species 25 might lead to a model that yields unreasonable results for other properties. This would especially be the case if the original error in the binding data was as a consequence of an incorrect free energy of solvation for one or more of the component species. Finding data to validate against is difficult though. One option might be to use gas phase quantum mechanical data. However, the charge ${ }_{30}$ distribution would be radically different from the condensed phase unless the ion pair is surrounded by at least the first solvation shell of water. An alternative choice is to use crystallographic data for solid forms as a reference point. Taking the calcium-aspartate interaction as our test case, there is a known crystal structure for $\mathrm{Ca}(\mathrm{L}-\mathrm{Asp}) \cdot 2 \mathrm{H}_{2} \mathrm{O},{ }^{75}$ which adopts an orthorhombic cell containing four formula 35 units. Two complications exist in using this as a reference structure. Firstly, only the heavy atom positions are reported as X-ray diffraction was used. This is easily resolved as the majority of the approximate hydrogen positions are unambiguous given the heavy atom geometry. Secondly, the structure contains the aspartate dianion, rather than the zwitterionic singly charged anion being considered in the 40 present study. To handle this, the charge of the $-\mathrm{NH}_{3}{ }^{+}$group was redistributed in the $-\mathrm{NH}_{2}$ moiety to achieve the correct overall charge and the $\mathrm{Ca}^{2+}-\mathrm{N}$ interaction was set equal to that of the calcium to oxygen of the carboxylate groups given that the

12 | [journal], [year], [vol], 00-00 
resulting charges of $\mathrm{N}$ and $\mathrm{O}$ are similar.

In the experimental crystal structure for $\mathrm{Ca}(\mathrm{L}-\mathrm{Asp}) \cdot 2 \mathrm{H}_{2} \mathrm{O}$, the calcium-oxygen bond lengths fall in the range of 2.429-2.608 $\AA$. Based on the original scaled force field parameters there is a systematic underestimation of these distances (2.324$52.540 \AA$ ), while using the parameters corrected against the ion pairing free energy the bond lengths $(2.439-2.601 \AA)$ are now in excellent agreement. Hence, while this is a somewhat indirect validation, it suggests that increasing the $\mathrm{Ca}-\mathrm{O}$ repulsive interaction for the ion pairs is indeed justified and likely to improve structural properties.

${ }_{10}$ Beyond just the overall free energy of ion pair formation, we can analyse the nature of the complete free energy landscape, as shown in Figure 3. Both aspartate and citrate show qualitatively similar free energy profiles, though they are quantitatively different. There is a minimum in which the anion binds in a monodentate configuration to calcium, at 3.5-3.6 $\AA$, with a point of inflection at 15 shorter distances corresponding to the bidentate arrangement. In effect, the bidentate form is a transition state for exchanging the oxygen atom of the organic that is coordinated to the calcium cation. Beyond this inner minimum there are discernable minima corresponding to solvent separated states, though the binding in these configurations is weaker than for the contact ion pair. Furthermore, the barrier to go 20 from the solvent separated minima to the directly coordinated state is generally less than ambient thermal energy and so there is little in the way of an activation barrier to contact ion pair formation. In line with experiment, we find that citrate exhibits much stronger binding to calcium than for aspartate, as would be expected from the charge of the anion being three times as large.

25 Acetate is found to have a quite different free energy profile of binding to that for aspartate and citrate with the curve exhibiting two distinct minima for the bi- and mono-dentate configurations. Although the monodentate form remains marginally more stable than the bidentate configuration, the free energy difference is small. In terms of rationalising this different behaviour for acetate relative to the other two 30 anions, the most probable factor is the different charge distribution for the carboxylate groups; in acetate the carboxylate is less polar than in aspartate or citrate, where the charges are similar. The rationale for this is that in the case of acetate it proved difficult to adopt the same degree of polarity while still obtaining both a reasonable free energy of solvation and a good description of water structure 35 around the carboxylate group.

For the case of aspartate, free energy profiles for ion pairing have been computed by Hamm et $a l^{37}$ for the binding with $\mathrm{Mg}^{2+}, \mathrm{Ca}^{2+}$ and $\mathrm{Sr}^{2+}$. In this prior work the binding free energy for aspartate to $\mathrm{Ca}^{2+}$ is found to be exothermic to the extent of $\sim 16 \mathrm{~kJ} / \mathrm{mol}$, making it more strongly bound than in the present study and further 40 from the experimental values.

As an additional qualitative validation of the results obtained with the conventional force field model, we have also performed simulations of the association of the organics with calcium ions using the reactive ReaxFF approach. Although obtaining quantitative free energies is more complex within this model, it ${ }_{45}$ does allow some of the assumptions regarding protonation state to be tested, while providing an indication of whether a totally different parameterisation and functional form of interaction would alter any of the behaviour observed for this system.

Considering first the case of acetate binding with $\mathrm{Ca}^{2+}$, the reactive model indicates that monodentate coordination by the carboxylate group is strongly

[journal], [year], [vol], 00-00 | 13 
preferred with a $\mathrm{Ca}-\mathrm{O}$ distance of $2.29 \AA$. Bidentate coordination appears to be predominantly a transition state during swapping of the oxygen that is bound to calcium, which occurs on 8-9 occasions during $2.5 \mathrm{~ns}$. Very similar behaviour is observed for aspartate binding to $\mathrm{Ca}^{2+}$. The fact that the reactive model shows 5 consistent modes of coordination in the ion pair for acetate and aspartate may suggest that the results from the conventional force field overestimate the presence of the bidentate configuration for acetate as a result of the charge distribution.

The ReaxFF simulations for citrate were run from several different initial configurations to probe the binding with the distinct functional groups. Some 10 starting geometries led to the ion pair dissociating, though the ions tended to remain in a solvent separated state rather than diffusing apart, consistent with the free energy profile computed with the conventional model. In other cases a carboxylate group that was not initially bound to calcium would enter its coordination environment such that the cation was chelated by either two carboxylate groups or 15 one carboxylate and the hydroxyl oxygen. Even when only one carboxylate group is directly attached to $\mathrm{Ca}^{2+}$, a second one is clearly hydrogen bonded to a water molecule from the first solvation shell of calcium.

Having considered the ion pairing between $\mathrm{Ca}^{2+}$ and the three organics alone, it is now possible to examine whether there may be some association between the 20 additives and stable pre-nucleation clusters. In our previous work we have shown that it is possible to simulate the formation of DOLLOP species through the use of a "brute force" approach, provided that the concentration is elevated to overcome the time scale for diffusion and collision of ions. DOLLOP formed in this way can then be examined at realistic concentrations in the presence of a background electrolyte, ${ }_{25}$ as per the experiments of Gebauer et $a l,{ }^{19}$ and be shown to be stable. ${ }^{20}$ Here we performed a limited number of simulations following this same strategy but now in the presence of organic anions. Unfortunately, the timescale accessible at present was insufficient to be able to identify any influence within the statistical uncertainty given that the binding of the organics is generally weaker than that of carbonate with 30 calcium. While some association between aspartate and citrate was observed with DOLLOP species, a more systematic approach is required to quantify any possible binding. That said, some interesting behaviour was seen for the case of aspartate, where the organic anions began to associate with each other to form a supramolecular polymer. While this can be ascribed to the particular concentration 35 of aspartate present in the simulations, we believe that the binding is not an artefact of the force field parameters for two reasons. First, the interaction distances found between two aspartate anions held together by two N-H--O hydrogen bonds in vacuo agree well between the force field and a quantum mechanical calculation at the $\mathrm{M} 06 / 6-31+\mathrm{G}^{* *}$ level (we note that any discrepancy in the distances would be ${ }_{40}$ reduced by improving the basis set quality further). Second, there is evidence from experiment that such association can and does occur under certain conditions. ${ }^{76}$

As a first attempt to shed some light on the possible binding of the three organics to DOLLOP-like species, we have quantified the thermodynamics of association with a single $\mathrm{CaCO}_{3}{ }^{(0)}$ ion pair. Unfortunately this is not as straight forward as for ${ }_{45}$ the previous ion pairing calculation because of the non-negligible probability that the ion pair will dissociate during the metadynamics simulation. To circumvent this problem we applied the following two-step procedure. First of all we performed a metadynamics simulation as above to construct a free energy profile for the collective variable $s$ subject to an artificial harmonic restraint $\left(K=2 \mathrm{eV} / \AA^{2}, r_{0}=3.4 \AA\right)$

14 | [journal], [year], [vol], 00-00 
between the $\mathrm{Ca}$ and carbonate carbon to keep the ion pair together. The resulting free energy profile was then used as a fixed bias for a set of 50 independent $5 \mathrm{~ns}$ unrestrained simulations, in which any deviations from a uniform sampling in $s$ result from differences between the restrained and unrestrained free energy 5 landscapes. During these simulations we calculated the probability distribution for the system along the biased collective variable and then extracted the free energy contribution due to the removed harmonic bond using;

$$
d A=-k_{B} T \ln (P(s))
$$

where $P(s)$ is the calculated probability distribution along $s$. This free energy 10 contribution was then added to the one previously calculated with metadynamics to obtain the total pairing free energy profile for the $\mathrm{CaCO}_{3}{ }^{(0)}$ ion pair to the organic molecules. This procedure did not completely eliminate the probability that the ionpair was broken during the calculations but it has the advantage that the parts of the trajectories not relevant to the calculations (when the $\mathrm{Ca}-\mathrm{Cc}$ distance was longer 15 than $4 \AA$ ) could be easily removed during the post processing. At large distances the effect of the restraint on the $\mathrm{Ca}-\mathrm{Cc}$ distance on the free energy profile is limited and this indeed was reflected by the fact that the probability distributions obtained during the second set of simulations was flat within thermal noise, as it should be once the free energy profile is exactly compensated by the metadynamics bias.

20 Results for the free energy of interaction between the three organic anions and the calcium carbonate ion pair in solution are given in Figure 4. In comparison to the binding of the organics to the $\mathrm{Ca}^{2+}$ ion alone, the free energy is less exothermic, as would be expected, due to the repulsion between the two anions that are within the first coordination sphere. What is less expected is that the effect is non-uniform and ${ }_{25}$ that citrate is the least affected, despite being the most highly charged anion. Clearly the presence of the aqueous environment screens the Coulomb repulsion between carbonate and more remote charged functional groups of the other ligand coordinated to calcium. In contrast, the electrostatic binding between $\mathrm{Ca}^{2+}$ and the more highly charged citrate is less screened since the interactions are through the 30 ligand, which has a much lower dielectric constant than water.

While citrate and aspartate maintain their similarity in having a minimum for direct coordination to the calcium of $\mathrm{CaCO}_{3}{ }^{(0)}$, the minimum free energy for acetate occurs at a longer distance intermediate between the contact and first solvent separated state for the $\mathrm{Ca}-\mathrm{Ace}^{+}$ion pair. Even though aspartate has a minimum that 35 is at a shorter distance than acetate, the strength of binding surprisingly goes as citrate $>>$ acetate $>$ aspartate. Based on this, it would be likely that acetate has more influence on ion pairing and perhaps DOLLOP formation than aspartate. However, the minimum in which aspartate coordinates to the calcium carbonate ion pair has a larger activation energy between it and the solvent separated state. Therefore 40 aspartate may exert a similar influence because of this kinetic factor. One thing is definitely clear from the simulation results - citrate is the only ligand that is likely to show significant association with pre-nucleation species in calcium carbonate solution.

[journal], [year], [vol], 00-00|15 


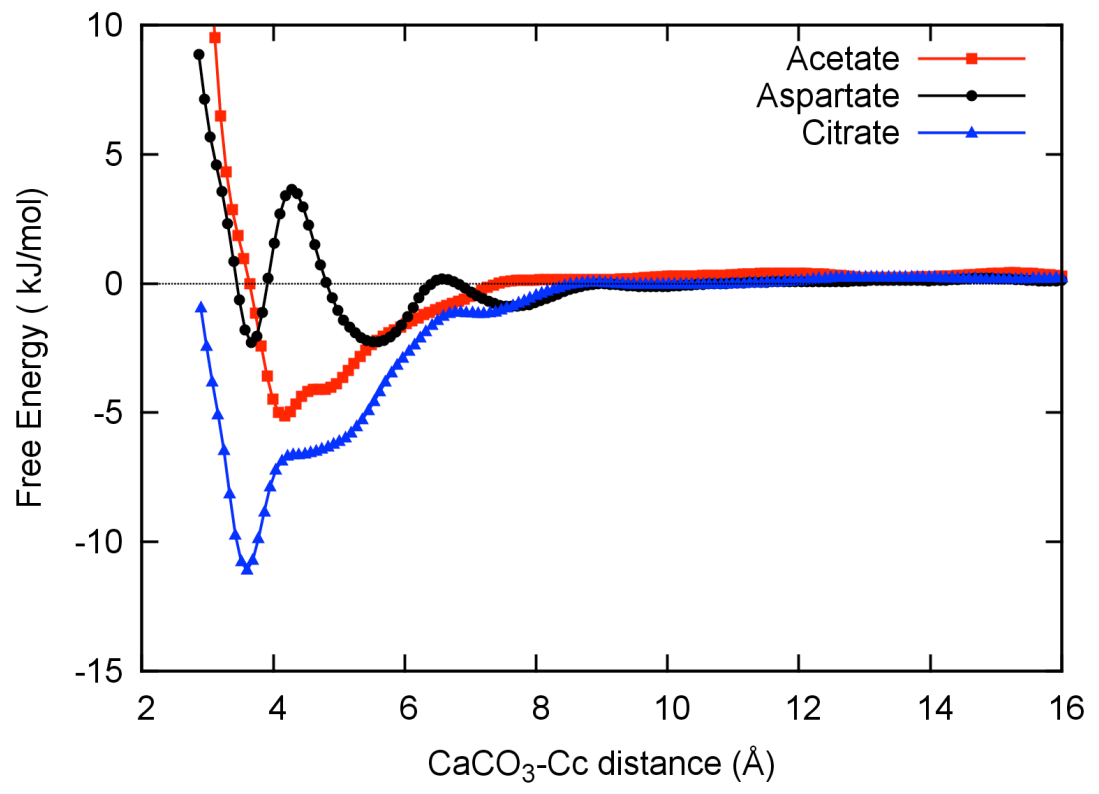

Figure 4 Free energy surface for association of the acetate, aspartate and citrate anions with the $\mathrm{CaCO}_{3}{ }^{(0)}$ ion pair in aqueous solution as a function of the minimum distance between $\mathrm{Ca}$ or $\mathrm{C}$ of carbonate $\left(\mathrm{CaCO}_{3}\right)$ and the carbon $(\mathrm{Cc})$ of the nearest carboxyl group of the organic species.

Reactive force field simulations have also been performed for the ion pair + organic systems described above. The most important finding was that the organic anions remained in the same protonation state as per the conventional model throughout the molecular dynamics in all cases. In order to verify that this was not 10 just an issue of timescale or an overly high barrier to protonation, a simulation was performed in which an excess proton was added to the simulation of the $\left(\mathrm{CaCO}_{3}\right.$ citrate $)^{3-}$ complex. Although the proton was initially part of a hydronium ion that was as far as possible from the ion pair within the simulation box, it rapidly diffuses via a Grotthus mechanism and leads to protonation of a carboxylate group of citrate.

${ }_{15}$ Hence the system appears to have sufficient opportunity to sample the protonation states during $2.5 \mathrm{~ns}$.

\section{D Binding of organics to calcium carbonate}

Aside from the influence of organic anions on pre-nucleation clusters of calcium ${ }_{20}$ carbonate, there is also the possibility that additives may alter the growth process via binding to post-nucleation species. In order to probe this possibility we have used metadynamics to determine the free energy profiles for binding of acetate, aspartate and citrate to two forms of post-nucleation calcium carbonate. First, given that under conditions relevant to biomineralisation the initial product is amorphous calcium ${ }_{25}$ carbonate, we have considered binding to a 36 formula unit ACC nanoparticle taken from our previous work. ${ }^{21}$ Since we predict that water content increases with increasing particle size, we have taken a nominally dry ACC nanoparticle for this smaller nanoparticle. Second, as the ultimate macroscopic product will be a

16 | [journal], [year], [vol], 00-00 
crystalline phase, we have also examined the binding of the organics to the basal $(10-14)$ surface of calcite as the dominant facet of the most stable phase.

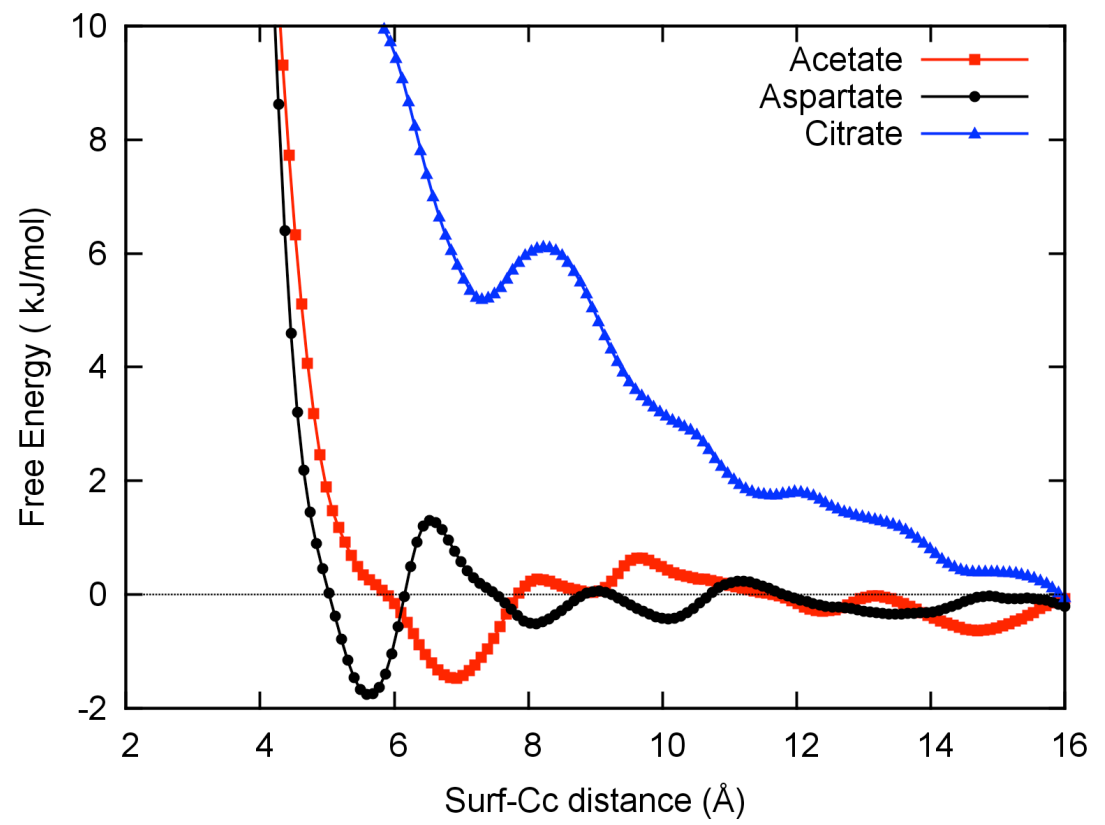

Figure 5 Free energy profile as a function of the normal distance above the calcite (10-14) surface to the nearest carbon of any carboxyl group of the three organics, acetate, aspartate and citrate. On the right hand side of the figure the free energy becomes asymptotic to zero, which is set as the free energy of the anion in bulk solution.

The resulting free energy curves for acetate, aspartate and citrate approaching the surface of the above two calcium carbonate materials are shown in Figures 5 and 6. ${ }_{10}$ Considering first the basal surface of calcite, all three ligands show slightly distinct behaviour. Acetate has a negligible degree of association with the basal surface of calcite as all minima are barely above the level of statistical noise. Experiments and simulations have both shown that there are two relatively strongly-ordered water layers over the basal face of calcite, ${ }^{77}$ and it appears that acetate is unable to ${ }_{15}$ penetrate this interfacial region. Aspartate on the other hand is initially slightly repelled by the surface, but can attain a solvent separated state that may be marginally stable at 5-6 $\AA$ above the surface. This parallels the behaviour previously seen for the carbonate anion. ${ }^{27}$ While the absolute depth of the minimum is no greater than for acetate, the barrier to exit the state is more pronounced. Finally, ${ }_{20}$ citrate exhibits no binding at the flat calcite surface as a result of the strong hydration experienced in aqueous solution.

Overall, it is found that none of the anions show any real association with the basal surface of calcite. This does not preclude any possible influence of additives on calcite growth, but we predict this would require either binding to steps or kink ${ }_{25}$ sites, or expression of other surfaces where calcium is more exposed. ${ }^{78}$ Indeed Elhadj et $a l^{79}$ have computed average binding enthalpies of over $400 \mathrm{~kJ} / \mathrm{mol}$ for the aspartate anion at steps on the calcite basal surface using semi-empirical cluster calculations in combination with a mix of explicit and continuum water models.

[journal], [year], [vol], 00-00 | 17 
Although we have yet to examine the influence of steps on binding with the present model, it appears unlikely that the free energy of binding of aspartate to calcite steps will be as exothermic as the above value based on the strength of interaction with the flat surface alone. Aside from the quantitative details, based on the indications 5 from the flat surface, it can be speculated that the order of influence for the additives considered would be aspartate $>$ acetate $>>$ citrate for processes involving calcite surfaces or interfacial environments where water is strongly ordered.

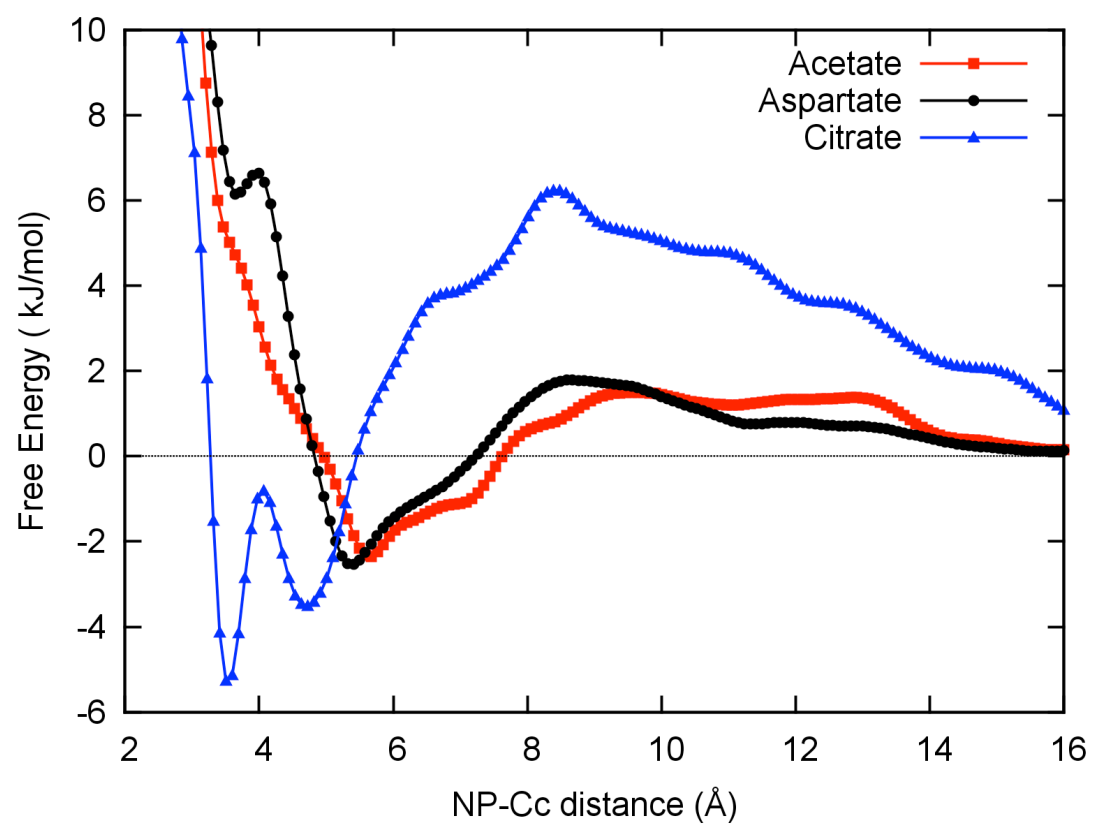

Figure 6 Free energy profile for bringing an organic anion from solution (right-hand side) towards a 36 formula unit nanoparticle (NP) (left-hand side) of dry amorphous calcium carbonate.

Turning now to consider the interaction of the three organics with ACC, a more complex situation is found to occur. Both acetate and aspartate exhibit a similar minimum that corresponds to a solvent separated complex with the nanoparticle. Although the bound minimum only has a slightly lower free energy than the anions 15 in bulk solution, there is a small activation barrier to separation from the nanoparticle, and so there is the possibility for acetate and aspartate to spend some time associated with ACC. In the case of citrate, the free energy profile of binding is radically different from that for the calcite surface, and to the profile for the other two organics. At first sight there is now a significantly exothermic state ${ }_{20}$ corresponding to citrate coordinating directly to the ACC nanoparticle. However, the unusual variation with distance indicates that something unexpected is occurring. Further analysis of the trajectories for the multiple walkers indicates that in some cases the citrate ligand pulls a calcium ion from the surface of ACC and so as it detaches the asymptotic limit is for formation of the $\mathrm{Ca}-(\mathrm{Cit})^{-}$ion pair. Associated ${ }_{25}$ with this fragmentation, some times a carbonate anion is also ejected from the ACC nanoparticle in order to remove the excess negative charge, as shown in Figure 7. Because of these observations we can deduce that the curve for citrate in Figure 6 is actually the convolution of the calcium-citrate ion pair and the underlying curve

18 | [journal], [year], [vol], 00-00 
actually being sought. This illustrates the difficulty of trying to map the free energy surface for complex systems in which they are alternative competing reactions to that of the collective variable under consideration. Part of the problem here may be because one of the smallest possible nanoparticles of ACC was employed as the 5 model and so the more open structure makes it easier for calcium ions to be removed by water in combination with the ligand. Although it has not been possible to quantify the interaction of citrate with ACC in the present work, it is also clear that citrate is not repelled by ACC in the same manner as the basal surface of calcite. The absence of a strongly ordered water layer makes it possible for citrate to 10 occasionally reach the surface of ACC and bind to calcium, though this sometimes leads to removal of the ion from the surface. Preliminary unbiased simulations for larger nanoparticles of ACC suggest that the tendency to remove calcium is a consequence of the small particle size for 36 formula units.
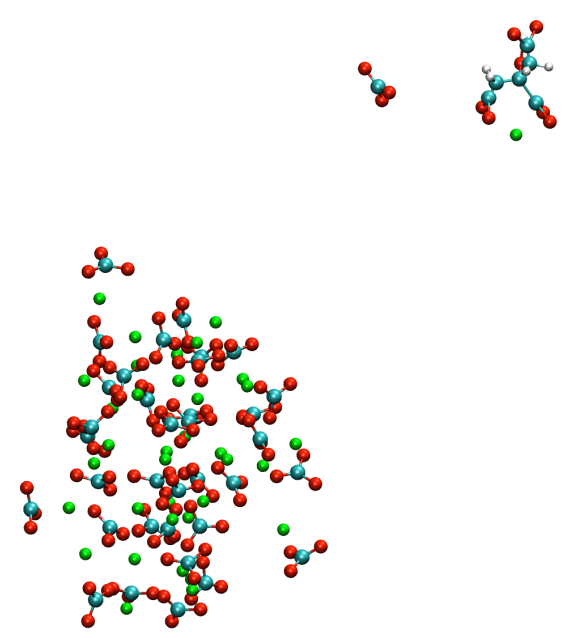

15 Figure 7 Configuration taken from the simulation of citrate in contact with an amorphous calcium carbonate nanoparticle initially containing 36 formula units. The citrate anion (top right) has detached taking a calcium cation with it. A carbonate ion (top centre right) then also dissociates from the nanoparticle. Surrounding water molecules are hidden for clarity. 


\section{E Influence of acetate, L-aspartate and citrate on in vitro precipitation}

A

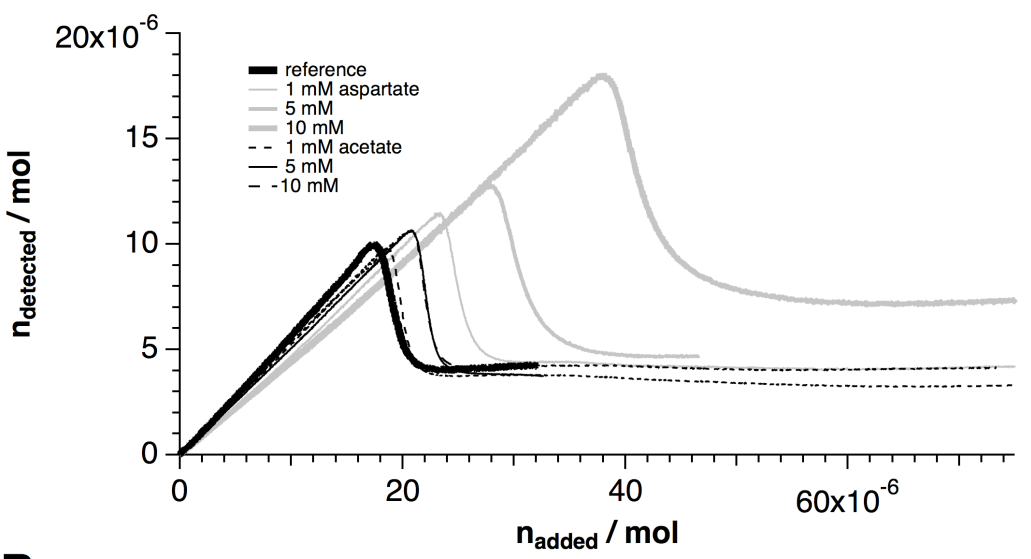

B

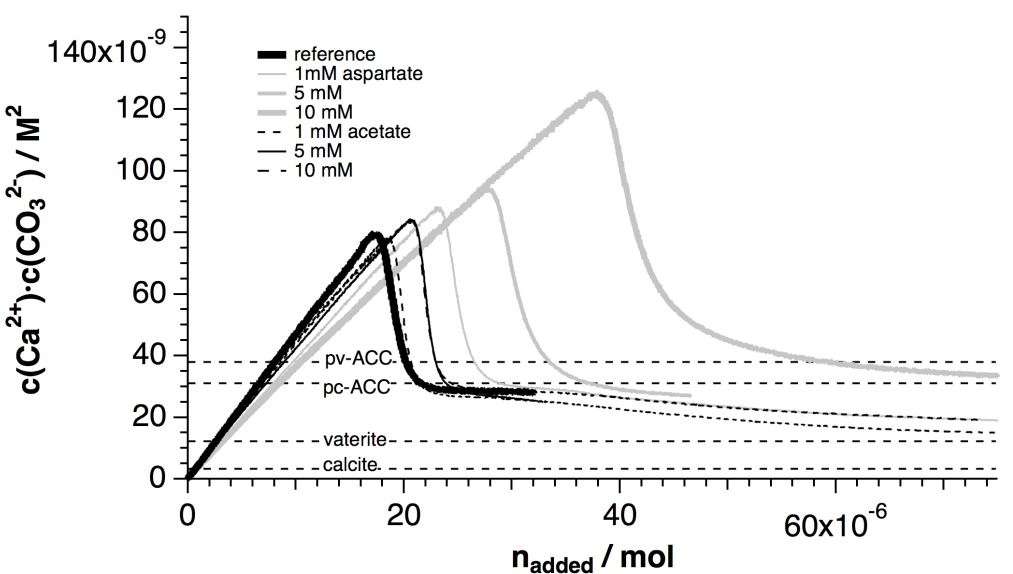

Figure 8. The influence of acetate and aspartate on the early stages of calcium carbonate precipitation. A: Development of the amount of free calcium in $50 \mathrm{~mL}$ carbonate buffer $(10 \mathrm{mM}, \mathrm{pH}$ $=9.00$ ) upon addition of dilute calcium chloride solution, in the absence (reference) and presence of different amounts of additives as indicated. The steep drop in the detected free calcium identifies the 10 nucleation event. B: Corresponding free ion product as a function of the amount of calcium added. Dashed lines mark the solubilities of proto-vaterite ACC (pv-ACC), proto-calcite-ACC (pc-ACC) ${ }^{18}$ vaterite, and calcite. ${ }^{18,80}$ Note that the curves of 5 and $10 \mathrm{mM}$ acetate largely overlap.

In order to assess the effect of the different additives on in vitro crystallisation, quantitative assays with and without the different molecular additives have been 15 conducted. The experiments were carried out at constant $\mathrm{pH}=9.00$, starting with pure carbonate buffer. Upon slow addition of dilute calcium solution into the buffer, supersaturation was generated until nucleation occurred, and solid forms of calcium carbonate were precipitated. This process was traced utilising a calcium ion selective

20 | [journal], [year], [vol], 00-00 
electrode and, as discussed in detail in previous work, ${ }^{23}$ the calcium curves can be used to quantitatively characterise the influence of additives on the early stages of calcium carbonate precipitation through comparison with the reference experiment carried out in the absence of additives. In principle, there are five different effects ${ }_{5}$ that can be identified and quantified based on the calcium curves; (i) complexation of calcium ions, which is apparent from a delayed increase in calcium; (ii) influence of the additives on the pre-nucleation equilibria, which is associated with an altered slope for the pre-nucleation calcium development; (iii) inhibition of nucleation can be identified by a shift of the maximum in the curves to later times (more addition of 10 calcium required) and to higher amounts of detected calcium; (iv) adsorption of the additives on nucleated particles can become apparent from a new increase of detected calcium after nucleation, i.e. an constant solubility is not maintained; (v) the solubility can be directly determined and shows whether the additives influence the type of amorphous phase or crystalline polymorph formed.

15 In the reference experiment (Figure $8 \mathrm{~A}$ ), the amount of free calcium in the carbonate buffer at first increases linearly upon constant addition of calcium chloride solution, while less calcium is detected than actually added. This is the prenucleation stage, and the calcium binding in combination with the $\mathrm{pH}$-titration can be used to quantitatively characterise the pre-nucleation cluster equilibrium. ${ }^{19}$ When 20 a critical point is reached, nucleation occurs and the amount of free calcium drops to a value that corresponds to the solubility of the precipitated phase (which is protocalcite ACC at this $\mathrm{pH}-$ level, and relates to calcite in terms of its short-range order $)^{18,19}$.

Titration curves obtained in the presence of 1,5 and $10 \mathrm{~mm}$ acetate show no ${ }_{25}$ distinct deviation from the reference experiment within the common limits of accuracy and reproducibility (Figure $8 \mathrm{~A}$ ). The slight tendency towards inhibition of nucleation seen at $10 \mathrm{~mm}$ (i.e. when acetate is equimolar to the carbonate buffer and thus is at a large excess over calcium carbonate species) cannot be regarded as significant (see ESI Figure S4). Indeed, similar observations were made when 10 $30 \mathrm{mM}$ sodium chloride was added to the buffer and therefore, if anything, corresponding effects are likely to originate from the higher salinity of the system (ESI Figure S4). At $1 \mathrm{~mm}$ L-aspartate, the delay of nucleation is somewhat more pronounced than at $10 \mathrm{~mm}$ acetate. With increasing concentration of aspartate, inhibition of nucleation becomes much more distinct and approximately twice the 35 amount of calcium has to be added at $10 \mathrm{~mm}$ aspartate for nucleation to occur. Furthermore, the level of free calcium after nucleation - which reflects the solubility of the precipitated calcium carbonate phase - appears to be higher than in the reference experiment (Figure $8 \mathrm{~A}$ ). This indicates that L-aspartate stabilises calcium carbonate phases that are less stable than those precipitated without 40 additives or in the presence of acetate. This is consistent with the findings of the simulations that aspartate has a stronger association with more disordered and solvated species (i.e. the stability of binding is $\mathrm{Ca}^{2+}>\mathrm{CaCO}_{3}{ }^{(0)}>\mathrm{ACC}>>$ calcite).

In addition to the above, we find that the pre-nucleation development in terms of the amount of free calcium ions (Figure $8 \mathrm{~A}$ ) is slightly flatter in both aspartate- and 45 acetate-containing samples than in the reference case. This effect can be attributed to a decrease in ionic activity caused by the charged additives, as evidenced by the fact that these differences become larger when the concentration of the additives is increased. Moreover, flattening of the pre-nucleation regime was also observed when sodium chloride was added to the buffer (cf. ESI Figure S4), and titrations of

[journal], [year], [vol], 00-00|21 
aqueous additive solutions without carbonate confirm their non-ideality by revealing a consistent influence of ionic strength on the measured calcium potential (see ESI Figure S5 \& Table S4). However, it is worth noting that these changes in the prenucleation slope may not exclusively be due to activity effects when it comes to the ${ }_{5}$ experiments in carbonate buffer. Comparing the data recorded for aspartate and acetate with those obtained in the presence of sodium chloride suggests that average slopes at a given concentration of aspartate and acetate are by trend smaller than those found for $\mathrm{NaCl}$ at the same ionic strength (see ESI Figure S6 \& Table S4). We note that the differences do only slightly exceed the common limits of experimental 10 error, while we regard the rather high number of experiments that would be required to achieve good statistical quantification of this minor effect unjustified. Therefore, we can only speculate that there may be a stabilising influence of these two additives on pre-nucleation clusters (apparent from the fact that more calcium is bound in clusters in their presence), which appears to be somewhat more distinct in the case 15 of aspartate. This indicates that the degree of cluster stabilisation increases with the number of carboxylate functional groups in the molecular additives, given that citrate shows a pronounced effect (see below). However, such a stabilising effect cannot be found for the polyacids. ${ }^{23,26}$ These findings agree with the results of the simulations for the association of the three organics with the $\mathrm{CaCO}_{3}{ }^{(0)}$ ion pair; 20 acetate and aspartate have at best a weak association with this species, while citrate definitely is capable of binding. Given that larger pre-nucleation clusters adopt the flexible DOLLOP structure that includes ion pair chains, we would expect the influence of the organics such species to be similar to that for the neutral ion pair, which accords with the experimental findings.

25 When dilution effects are corrected for by calculation of actual free ion products (Figure $8 \mathrm{~B}$ ), it becomes evident that in the reference experiment, as well as in the presence of acetate, proto-calcite ACC (pc-ACC) is initially nucleated, in agreement with earlier findings. ${ }^{19,23}$ During the early post-nucleation stage, this metastable intermediate appears to transform into more stable species, as the calculated ion ${ }_{30}$ product gradually approaches the solubility of vaterite (note that the concurrent formation of more stable species such as calcite is not necessarily reflected by the measured solubility, which is governed by the most soluble phase, because the system has not reached equilibrium according to Gibbs' phase rule). X-ray diffraction analyses of precipitates isolated after completed titration show that ${ }_{35}$ calcite and vaterite co-exist in all samples, at varying relative amounts (data not shown). We could not discern any clear trend that would indicate polymorph selection. Only in the case of aspartate, a distinct influence on the stability of the initially precipitated phase can be detected (Figure $8 \mathrm{~B}$ ). At 5 and $10 \mathrm{~mm}$ aspartate, the free ion product decreases very slowly to the solubility value corresponding to

${ }_{40}$ pc-ACC after nucleation: again, this suggests that more soluble (i.e. less stable) amorphous phases are stabilised by the additive. It remains unclear, though, whether (energetically downhill) transitions between different forms of ACC occur, or interactions of ACC with the additive impede the establishment of a constant solubility product via inhibition of growth (e.g. by adsorption onto nucleated 45 particles).

The influence of citrate ions on the early stages of calcium carbonate crystallisation has been discussed already in a previous study, ${ }^{26}$ and we summarise the main observations here. First, it was found that citrate and calcium ions associate in solution (complexation), which is the reason for the common application of citrate

22 | [journal], [year], [vol], 00-00 
as an antiscalant. This effect became manifest through a distinct upward bend of the pre-nucleation curve prior to becoming asymptotically linear, as a result of complexation of calcium by citrate retarding the onset of pre-nucleation cluster formation by reducing the concentration of free calcium. ${ }^{23,26}$ In the present work, 5 such association equilibrium between calcium and acetate, as well as aspartate, could neither be detected in carbonate buffers (Figure $8 \mathrm{~A}$ ) nor in pure water (ESI Figure S5 and Table S4). Experiments with citrate further revealed a distinct flattening of the pre-nucleation slope already at very low concentrations $(50 \mathrm{mg} / \mathrm{L}$, corresponding to ca. $0.26 \mathrm{mM}$ ), which was much more pronounced than what has

10 been observed for $10 \mathrm{mM}$ acetate and aspartate in this work. Although citrate carries three carboxylate groups that all are completely deprotonated at the investigated $\mathrm{pH}$ level, simple activity effects can be neglected due to the small concentration of additive used. Therefore, the flatter slope in the pre-nucleation stage indicated that more calcium was bound in clusters than in the absence of citrate or, in other words,

15 that the clusters were stabilised by the additive. Quantitative evaluation suggested that this stabilisation was ca. 1-2 $\mathrm{kJ} \mathrm{mol}^{-1}$ (relative to the additive-free case and in terms of the free enthalpy change for the formation of a $\mathrm{CaCO}_{3}$ unit within a prenucleation cluster). ${ }^{26}$ In contrast to aspartate, citrate was shown to only slightly inhibit nucleation, mainly as a consequence of calcium ion complexation, and 20 moreover did not stabilise nucleated particles to any noticeable extent. In turn, it appeared to control the polymorphism of the final solid product, since pure-phase calcite was found in presence of citrate. ${ }^{26}$

\section{Discussion}

Surprisingly, the investigated molecular additives show quite marked differences ${ }_{25}$ with respect to their influence on the early stages of in vitro calcium carbonate crystallisation, although they essentially differ merely by the number of charged carboxylate functions (at $25^{\circ} \mathrm{C}$ : acetate, $\mathrm{pK}_{\mathrm{a}}=4.76$; L-aspartate, $\mathrm{pK}_{\mathrm{a}}(\alpha-\mathrm{COOH})=$ $1.95 ; \mathrm{pK}_{\mathrm{a}}(\beta-\mathrm{COOH})=3.71 ;$ citrate, $\left.\mathrm{pK}_{\mathrm{a}}(1)=3.13, \mathrm{pK}_{\mathrm{a}}(2)=4.76, \mathrm{pK}_{\mathrm{a}}(3)=6.40\right) .{ }^{81}$ In terms of our recently introduced categorisation of additives, ${ }^{23} \mathrm{~L}$-aspartate can be 30 classified as a type III (inhibition of nucleation) and possibly also weak type II additive (stabilisation of pre-nucleation clusters). The latter could also apply for acetate, which otherwise did not show any significant effect. Citrate belongs to types I/II/III/V (complexation of calcium, stabilisation of pre-nucleation clusters, inhibition of nucleation, polymorph selection toward calcite). Interestingly, while ${ }_{35}$ both aspartate and citrate are capable of inhibiting nucleation, this effect is not necessarily based on ion complexation, as classically assumed. The titration data recorded in the present work demonstrate that aspartate does not form complexes with calcium under the given experimental conditions, whereas citrate-calcium association is evident already at the lowest concentrations investigated. ${ }^{26}$ Finally, 40 our results suggest that of the three additives considered here only aspartate can stabilise intermediate phases. In this regard, already monomeric L-aspartate appears to exhibit additive properties that are related to its macromolecular analogue, poly(aspartic acid), which has proven to be capable of stabilising an unstable intermediate $^{26}$ that presumably corresponds to PILP. ${ }^{25}$ The atomistic basis ${ }_{45}$ underlying the substantially different effects exerted by these rather simple molecules is at first puzzling.

The experimental finding that acetate does not interact with the species present

[journal], [year], [vol], 00-00|23 
during the early stages of precipitation is largely in accord with the computer simulations. When it comes to interactions with the surface of ACC, the simulations do not show a distinct difference between acetate and aspartate since both only show a weak solvent separated association. Given that the strongest difference in $s$ behaviour between acetate and aspartate occurs when they bind to calcium, either alone or in an ion pair (or potentially DOLLOP through association with other ion pairs), this suggests that the stabilisation of nucleated amorphous nanoparticles may be based upon additional interaction with other species. In previous work ${ }^{21}$ it was shown that ion pairs of calcium carbonate readily bind to the surface of ACC 10 nanoparticles, and so it may be possible that calcium-organic ion pairs may also do so. Because the association of aspartate with $\mathrm{Ca}^{2+}$ (aq) is stronger than for acetate, while it is the opposite for $\mathrm{CaCO}_{3}{ }^{(0)}$, this suggests that the altered stabilisation of amorphous nanoparticles is more likely to occur through attachment of calciumorganic ion pairs to the nanoparticle surface.

15 The simulations show that citrate forms complexes with calcium ions, and moreover indicate that it interacts more strongly than the other additives with prenucleation clusters, but not with the ordered surfaces of crystalline forms of calcium carbonate. The strong interaction of citrate with pre-nucleation clusters may rationalize why the clusters are experimentally found to be stabilised, while the 20 weaker interaction with aspartate might be reflected in the experimental findings as well. Experimentally, both aspartate and citrate do interact with pre-nucleation clusters and amorphous nanoparticles, however the effects are distinct (aspartate: inhibition of nucleation and stabilisation of amorphous forms; citrate: minor inhibition of nucleation, mostly based on a lowered level of supersaturation due to 25 complexation of ions). That the inhibition of nucleation and the stabilisation of amorphous intermediates would be more efficient at intermediate strength of interactions is counter-intuitive. Since the mechanism of calcium carbonate nucleation has yet to be determined within the simulations, it is difficult to unambiguously explain this effect based on atomistic considerations. However, there 30 are at least two possible hypotheses. Firstly, citrate binds more strongly to prenucleation species than post-nucleation forms, while aspartate binds with a similar exothermicity to all species. Hence the concentration of aspartate on ACC nanoparticles may be higher due to less competition. Secondly, since nucleation of amorphous calcium carbonate nanoparticles appears to proceed via aggregation of ${ }_{35}$ pre-nucleation clusters, ${ }^{82}$ aspartate may be effective through colloidal stabilisation. Binding of the additives to DOLLOPs may render their aggregation to yield larger ACC nanoparticles difficult. In this context it is important to note that aspartate appears to be distinct from citrate as it readily assembles into supramolecular polymers too. These species may be much more efficient in colloidal stabilisation 40 than monomers, perhaps in coarse analogy to the steric stabilisation of nanoparticle dispersions by polymers. The tendency of aspartate to form clusters may moreover explain the fact that it can stabilise amorphous nanoparticles efficiently. However, it should be noted that the mere occurrence of clusters of an organic additive is not necessarily connected to a clear and distinct influence on the early stages of calcium ${ }_{45}$ carbonate crystallisation. The geometry and energetics of all possible interactions (that is, with ions, ion pairs, DOLLOP, ACC and crystalline forms) are expected to be different and dependent on the particular additive. This is comparable to the situation for "classical" polymers that are joined by covalent bonds; polymeric additives with the same chemical function but different backbones can have distinct

24 | [journal], [year], [vol], 00-00

This journal is @ The Royal Society of Chemistry [year] 
"fingerprints" during the early stages of calcium carbonate crystallisation, for instance, poly(aspartic acid) and poly(acrylic acid). ${ }^{23,26}$ Thus it is also possible that certain supramolecular polymers of organic additives do not exhibit any influence on the early stages of precipitation of calcium carbonate at all. Of the two hypotheses 5 mentioned above, the simulation data is consistent with the first proposal in showing that competition between species may result in the selectivity for post-nucleation species, while the second hypothesis has yet to be tested theoretically.

Last, but not least, it may be speculated that the ability of citrate to interact with all species that occur during the early stages of calcium carbonate crystallisation is 10 the basis of polymorph selection toward calcite by this additive; the interactions may lead to the stabilisation of proto-calcite ${ }^{18}$ structural forms throughout the different stages of precipitation. Indeed the scenario here may have parallels with that proposed by Freeman $e t a l^{22}$ for the egg shell protein ovocledin-17 and ACC. Citrate initially interacts favourably with the pre-nucleation clusters and perhaps the early

15 amorphous phases, and may preferentially alter their stability. However, when nucleation to the crystalline phase occurs then citrate would be expelled since it fails to bind to ordered flat surfaces. Given the lack of binding to the majority of the basal surface of calcite (i.e. away from steps and kinks) substantial energy would be released through the dissolution of citrate from this polymorph that might drive its 20 formation.

PR, RD and JDG thank the Australian Research Council and Curtin University for funding, as well as iVEC and NCI for the provision of computer time. MK appreciates funding of a postdoc position by BASF SE. DG thanks Markus ${ }_{25}$ Antonietti for a loan titration system, and Helmut Cölfen for his support. TRW and LBW acknowledge the EPSRC Programme Grant 'Hard-Soft Interfaces: From Understanding to Engineering' (EP/I001514/1), and HECToR, the national highperformance computing facility of the U.K. LBW thanks the EPSRC for PhD support via a DTA grant.

\section{${ }_{30}$ References}

${ }^{a}$ Nanochemistry Research Institute/Department of Chemistry, Curtin University, PO Box U1987, Perth,WA 6845, Australia.Fax: 619266 4699; Tel: 619266 7800; E-mail: J.Gale@curtin.edu.au ${ }^{b}$ Department of Physical Chemistry, University of Konstanz, Universitätsstrasse 10, Box 714, D78457 Konstanz, Germany Fax: 49 7531 883898; Tel: 497531 882169; E-mail:

35 Denis.Gebauer@uni-konstanz.de

${ }^{c}$ Department of Physics/Centre for Scientific Computing, University of Warwick, Gibbet Hill Road, Coventry, CV4 7AL, UK. Fax: 442476 573133; Tel: 4424765 74580; E-mail: d.quigley@warwick.ac.uk

${ }^{d}$ Department of Chemistry/Centre for Scientific Computing, University of Warwick, Gibbet Hill

40 Road, Coventry, CV4 7AL, UK. Fax: 442476 524112; Tel: 442476 523653; E-mail: louise.wright@warwick.ac.uk

${ }^{e}$ Institute for Frontier Materials, Deakin University, Geelong, Vic, 3217, Australia. Fax: 613522

71103; Tel: 613522 73116; E-mail: tiffany.walsh@deakin.edu.au

$\dagger$ Electronic Supplementary Information (ESI) available: Parameters for conventional unreactive force field (Tables S1, S2, S3); schematics of anions showing atom types and charges for the conventional force field (Figures S1-S3); reproducibility of the titration experiments (Figure S4); 50 evolution of the amount of free calcium upon dosing calcium chloride into aqueous additive solutions at different concentrations (Figure S5, Table S4); effect of the different additives on the

[journal], [year], [vol], 00-00|25 
slope of the increase during the pre-nucleation stage in carbonate buffer (Figure S6). See DOI: $10.1039 / \mathrm{b} 000000 \mathrm{x} /$

1. H. Lowenstam and S. Weiner, On Biomineralization, Oxford University Press, New York, 1989.

2. L. Addadi, D. Joester, F. Nudelman, and S. Weiner, Chem. Eur. J., 2006, 12, 980-987.

3. J. Aizenberg, G. Lambert, S. Weiner, and L. Addadi, J. Am. Chem. Soc., 2002, 124, 32-39.

4. J. Aizenberg, A. Tkachenko, S. Weiner, L. Addadi, and G. Hendler, Nature, 2001, 412, 819822.

10 5. F. C. Meldrum and H. Cölfen, Chem. Rev., 2008, 108, 4332-4432.

6. L. B. Gower, Chem. Rev., 2008, 108, 4551-4627.

7. N. A. J. M. Sommerdijk and H. Cölfen, MRS Bull., 2010, 35, 116-121.

8. C. J. Stephens, S. F. Ladden, F. C. Meldrum, and H. K. Christenson, Adv. Funct. Mater., 2010, 20, 2108-2115.

15 9. R.-Q. Song and H. Cölfen, CrystEngComm, 2011, 13, 1249-1276.

10. C. C. Tang, S. P. Thompson, J. E. Parker, A. R. Lennie, F. Azough, and K. Kato, J. Appl. Crystallogr., 2009, 42, 225-233.

11. M. Neumann and M. Epple, Eur. J. Inorg. Chem., 2007, 2007, 1953-1957.

12. J. Wang and U. Becker, Am. Mineral., 2009, 94, 380-386.

20 13. R. Demichelis, P. Raiteri, J. D. Gale, and R. Dovesi, CrystEngComm, 2012, 14, 44-47.

14. L. Addadi, S. Raz, and S. Weiner, Adv. Mater., 2003, 15, 959-970.

15. A. V. Radha, T. Z. Forbes, C. E. Killian, P. U. P. A. Gilbert, and A. Navrotsky, Proc. Natl. Acad. Sci. U.S.A., 2010, 107, 16438-16443.

16. E. Loste, R. M. Wilson, R. Seshadri, and F. C. Meldrum, J. Cryst. Growth, 2003, 254, 206-218.

25 17. R. S. K. Lam, J. M. Charnock, A. Lennie, and F. C. Meldrum, CrystEngComm, 2007, 9, 1226 1236.

18. D. Gebauer, P. N. Gunawidjaja, J. Y. P. Ko, Z. Bacsik, B. Aziz, L. J. Liu, Y. F. Hu, L. Bergström, C. W. Tai, T. K. Sham, M. Edén, and N. Hedin, Angew. Chem. Int. Ed., 2010, 49, $8889-8891$.

30 19. D. Gebauer, A. Völkel, and H. Cölfen, Science, 2008, 322, 1819-1822.

20. R. Demichelis, P. Raiteri, J. D. Gale, D. Quigley, and D. Gebauer, Nat. Commun., 2011, 2, 590.

21. P. Raiteri and J. D. Gale, J. Am. Chem. Soc., 2010, 132, 17623-17634.

22. C. L. Freeman, J. H. Harding, D. Quigley, and P. M. Rodger, Angew. Chem. Int. Ed., 2010, 49, $5135-5137$.

35 23. D. Gebauer, H. Cölfen, A. Verch, and M. Antonietti, Adv. Mater., 2009, 21, 435-439.

24. D. Gebauer, A. Verch, H. G. Börner, and H. Cölfen, Cryst. Growth Des., 2009, 9, 2398-2403.

25. L. B. Gower and D. J. Odom, J. Cryst. Growth, 2000, 210, 719-734.

26. A. Verch, D. Gebauer, M. Antonietti, and H. Cölfen, Phys. Chem. Chem. Phys., 2011, 13, 16811-16820.

40 27. P. Raiteri, J. D. Gale, D. Quigley, and P. M. Rodger, J. Phys. Chem. C, 2010, 114, 5997-6010.

28. Y. Wu, H. L. Tepper, and G. A. Voth, J. Chem. Phys., 2006, 124, 024503.

29. J. A. Griffiths and D. M. Heyes, Langmuir, 1996, 12, 2418-2424.

30. P. Dauber-Osguthorpe, V. A. Roberts, D. J. Osguthorpe, J. Wolff, M. Genest, and A. T. Hagler, Proteins Struct. Funct. Bioinf., 1988, 4, 31-47.

45 31. N. H. de Leeuw and T. G. Cooper, Cryst. Growth Des., 2004, 4, 123-133.

32. U. Aschauer, D. Spagnoli, P. Bowen, and S. C. Parker, J. Colloid Interface Sci., 2010, 346, 226-231.

33. S. L. Mayo, B. D. Olafson, and W. A. Goddard, J. Phys. Chem., 1990, 94, 8897-8909.

34. D. M. Duffy and J. H. Harding, J. Mater. Chem., 2002, 12, 3419-3425.

50 35. D. Quigley, P. M. Rodger, C. L. Freeman, J. H. Harding, and D. M. Duffy, J. Chem. Phys., 2009, 131, 094703.

36. B. R. Brooks, R. E. Bruccoleri, B. D. Olafson, D. J. States, S. Swaminathan, and M. Karplus, J. Comp. Chem., 1983, 4, 187-217.

37. L. M. Hamm, A. F. Wallace, and P. M. Dove, J. Phys. Chem. B, 2010, 114, 10488-10495.

55 38. R. A. Metzler, G. A. Tribello, M. Parrinello, and P. U. P. A. Gilbert, J. Am. Chem. Soc., 2010, 132, $11585-11591$.

39. W. D. Cornell, P. Cieplak, C. I. Bayly, I. R. Gould, K. M. Merz, D. M. Ferguson, D. C. Spellmeyer, T. Fox, J. W. Caldwell, and P. A. Kollman, J. Am. Chem. Soc., 1995, 117, 51795197.

26 | [journal], [year], [vol], 00-00 
40. C. L. Freeman, J. H. Harding, D. J. Cooke, J. A. Elliott, J. S. Lardge, and D. M. Duffy, J. Phys. Chem. B, 2007, 111, 11943-11951.

41. A. Pavese, M. Catti, S. C. Parker, and A. Wall, Phys. Chem. Miner., 1996, 23, 89-93.

42. Y. Zhao and D. G. Truhlar, Theor. Chem. Acc., 2007, 120, 215-241.

5 43. A. V. Marenich, R. M. Olson, C. P. Kelly, C. J. Cramer, and D. G. Truhlar, J. Chem. Theory Comput., 2007, 3, 2011-2033.

44. Y. Shao, L. F. Molnar, Y. Jung, J. Kussmann, C. Ochsenfeld, S. T. Brown, A. T. B. Gilbert, L. V. Slipchenko, S. V. Levchenko, D. P. O’Neill, R. A. DiStasio Jr, R. C. Lochan, T. Wang, G. J. O. Beran, N. A. Besley, J. M. Herbert, C. Yeh Lin, T. Van Voorhis, S. Hung Chien, A. Sodt, R.

10 P. Steele, V. A. Rassolov, P. E. Maslen, P. P. Korambath, R. D. Adamson, B. Austin, J. Baker, E. F. C. Byrd, H. Dachsel, R. J. Doerksen, A. Dreuw, B. D. Dunietz, A. D. Dutoi, T. R. Furlani, S. R. Gwaltney, A. Heyden, S. Hirata, C.-P. Hsu, G. Kedziora, R. Z. Khalliulin, P. Klunzinger, A. M. Lee, M. S. Lee, W. Liang, I. Lotan, N. Nair, B. Peters, E. I. Proynov, P. A. Pieniazek, Y. Min Rhee, J. Ritchie, E. Rosta, C. David Sherrill, A. C. Simmonett, J. E. Subotnik, H. Lee

15 Woodcock III, W. Zhang, A. T. Bell, A. K. Chakraborty, D. M. Chipman, F. J. Keil, A. Warshel, W. J. Hehre, H. F. Schaefer III, J. Kong, A. I. Krylov, P. M. W. Gill, and M. HeadGordon, Phys. Chem. Chem. Phys., 2006, 8, 3172.

45. W. Smith, Mol. Simul., 2006, 32, 933-933.

46. W. Smith and I. T. Todorov, Mol. Simul., 2006, 32, 935-943.

20 47. K. Vanommeslaeghe, E. Hatcher, C. Acharya, S. Kundu, S. Zhong, J. Shim, E. Darian, O. Guvench, P. Lopes, I. Vorobyov, and A. D. Mackerell, J. Comput. Chem., 2010, 31, 671-690.

48. http://dogmans.umaryland.edu/ kenno/cgenff/index.html, 2012.

49. A. C. T. van Duin, S. Dasgupta, F. Lorant, and W. A. Goddard, J. Phys. Chem. A, 2001, 105, 9396-9409.

25 50. J. D. Gale and A. L. Rohl, Mol. Simul., 2003, 29, 291-341.

51. J. D. Gale, P. Raiteri, and A. C. T. van Duin, Phys. Chem. Chem. Phys., 2011, 13, 16666.

52. H. Manzano, R. J. M. Pellenq, F.-J. Ulm, M. J. Buehler, and A. C. T. van Duin, Langmuir, 2012, 120222072349005.

53. O. Rahaman, A. C. T. van Duin, W. A. Goddard, and D. J. Doren, J. Phys. Chem. B, 2011, 115, $30 \quad 249-261$.

54. G. Bussi, D. Donadio, and M. Parrinello, J. Chem. Phys., 2007, 126, 014101.

55. P. Raiteri, J. D. Gale, and G. Bussi, J. Phys. Condens. Matter, 2011, 23, 334213.

56. S. Plimpton, J. Comp. Phys., 1995, 117, 1-19.

57. M. Bonomi, D. Branduardi, G. Bussi, C. Camilloni, D. Provasi, P. Raiteri, D. Donadio, F.

35 Marinelli, F. Pietrucci, R. A. Broglia, and M. Parrinello, Comput. Phys. Commun., 2009, 180, 1961-1972.

58. A. Barducci, G. Bussi, and M. Parrinello, Phys. Rev. Lett., 2008, 100, 020603.

59. P. Raiteri, A. Laio, F. L. Gervasio, C. Micheletti, and M. Parrinello, J. Phys. Chem. B, 2006, 110, 3533-3539.

40 60. CPMD, IBM Corp. and Max-Planck-Institut für Festkörperforschung Stuttgart, 2000.

61. A. D. Becke, Phys. Rev. A, 1988, 38, 3098-3100.

62. C. T. Lee, W. T. Yang, and R. G. Parr, Phys. Rev. B, 1988, 37, 785-789.

63. D. Vanderbilt, Phys. Rev. B, 1990, 41, 7892-7895.

64. D. Gao, P. Svoronos, P. K. Wong, D. Maddalena, J. Hwang, and H. Walker, J. Phys. Chem. A, 2005, 109, 10776-10785.

65. E. C. Meng, P. Cieplak, J. W. Caldwell, and P. A. Kollman, J. Am. Chem. Soc., 1994, 116, 12061-12062.

66. P. I. Nagy and B. Noszál, J. Phys. Chem. A, 2000, 104, 6834-6843.

67. W. Sang-Aroon and V. Ruangpornvisuti, Int. J. Quant. Chem., 2008, 108, 1181-1188.

50 68. J. M. Wang, W. Wang, S. H. Huo, M. Lee, and P. A. Kollman, J. Phys. Chem. B, 2001, 105, 5055-5067.

69. A. M. Toth, M. D. Liptak, D. L. Phillips, and G. C. Shields, J. Chem. Phys., 2001, 114, 4595.

70. R. G. Pearson, J. Am. Chem. Soc., 1986, 108, 6109-6114.

71. M. V. Fedotova and S. E. Kruchinin, J. Mol. Liq., 2011, 164, 201-206.

55 72. J. Wang, G. Román-Pérez, J. M. Soler, E. Artacho, and M.-V. Fernández-Serra, J. Chem. Phys., 2011, 134, 024516.

73. E. Shock and C. M. Koretsky, Geochim. Cosmochim. Acta, 1995, 59, 1497-1532.

74. A. K. Covington and E. Y. Danish, J. Solution Chem., 2009, 38, 1449-1462.

75. H. Schmidbaur, I. Bach, D. L. Wilkinson, and G. Müller, Chem. Ber., 1989, 122, 1439-1444.

[journal], [year], [vol], 00-00|27 
76. M. Kellermeier, R. Rosenberg, A. Moise, U. Anders, M. Przybylski, and H. Cölfen, Faraday Discuss., 2012, DOI: 10.1039/C2FD20060K.

77. P. Geissbühler, P. Fenter, E. DiMasi, G. Srajer, L. B. Sorensen, and N. C. Sturchio, Surf. Sci., 2004, 573, 191-203.

5 78. H. H. Teng and P. M. Dove, Am. Mineral., 1997, 82, 878-887.

79. S. Elhadj, E. A. Salter, A. Wierzbicki, J. J. De Yoreo, N. Han, and P. M. Dove, Cryst. Growth Des., 2006, 6, 197-201.

80. L. Brečević and A. E. Nielsen, J. Cryst. Growth, 1989, 98, 504-510.

81. D. R. Lide, CRC handbook of chemistry and physics : a ready-reference book of chemical and physical data : 2002-2003, CRC Press, Boca Raton, 2002.

82. D. Gebauer and H. Cölfen, Nano Today, 2011, 6, 564-584.

28 | [journal], [year], [vol], 00-00

This journal is ( ) The Royal Society of Chemistry [year] 